This is the peer reviewed version of the following article:

Mirjolet M., Vasili H.B., López-Conesa L., Estradé S., Peiró F., Santiso J., Sánchez F., Machado P., Gargiani P., Valvidares M., Fontcuberta J.. Independent Tuning of Optical Transparency Window and Electrical Properties of Epitaxial SrVO3 Thin Films by Substrate Mismatch. Advanced Functional Materials, (2019). 29. 1904238: - . 10.1002/adfm.201904238,

which has been published in final form at https://dx.doi.org/10.1002/adfm.201904238. This article may be used for non-commercial purposes in accordance with Wiley Terms and Conditions for Use of Self-Archived Versions. 


\title{
Independent Tuning of Optical Transparency Window and Electrical Properties of Epitaxial SrVO3 Thin Films by Substrate Mismatch
}

\author{
Mathieu Mirjolet, Hari Babu Vasili, LLuís López-Conesa, Sònia Estradé, Francesca Peiró, \\ José Santiso, Florencio Sánchez, Pamela Machado, Pierluigi Gargiani, Manuel Valvidares \\ and Josep Fontcuberta*
}

M. Mirjolet, Dr. F. Sánchez, P. Machado, Prof. J. Fontcuberta

Institut de Ciència de Materials de Barcelona (ICMAB-CSIC), Campus UAB, Bellaterra

08193, Catalonia, Spain

E-mail: fontcuberta@icmab.cat

Dr. H. B. Vasili, Dr. P. Gargiani, Dr. M. Valvidares

ALBA Synchrotron Light Source, Cerdanyola del Vallès 08290, Catalonia, Spain

Dr. L. López-Conesa, Dr. S. Estradé, Dr. F. Peiró

Department of Electronics and Biomedical Engineering, University of Barcelona. C/ Martí i Franquès 1, Barcelona 08028, Catalonia, Spain

Dr. L. López-Conesa, Dr. S. Estradé, Dr. F. Peiró

Centres Científics i Tecnològics de la Universitat de Barcelona (CCiT-UB). C/ Lluís Solé i

Sabarís s/n, Barcelona 08028, Catalonia, Spain

Dr. L. López-Conesa

Institute of Nanoscience and Nanotechnology of the University of Barcelona ( $\operatorname{In}^{2} \mathrm{UB}$ ). C/

Martí i Franquès 1, Barcelona 08028, Catalonia, Spain

Dr. J. Santiso

Institut Català de Nanociència i Nanotecnologia (ICN2 - CSIC \& BIST), Campus UAB, Bellaterra 08193, Catalonia, Spain

Keywords: correlated metal oxides, orbital occupancy, plasma frequency, strontium vanadate, transparent conducting oxides

Transparent metallic oxides are pivotal materials in information technologies, photovoltaics or even in architecture. They display the rare combination of metallicity and transparency in the visible range because of weak interband photon absorption and weak screening of free carriers to impinging light. However, the workhorse of current technology, indium tin oxide (ITO), is facing severe limitations and alternative approaches are needed. $\mathrm{AMO}_{3}$ perovskites, $\mathrm{M}$ being a nd ${ }^{1}$ transition metal, and $\mathrm{A}$ an alkaline-earth, have a genuine metallic character and, in contrast to conventional metals, the electron-electron correlations within the $\mathrm{nd}^{1}$ band enhance 
the carriers effective mass $\left(\mathrm{m}^{*}\right)$ and bring the transparency window limit (marked by the plasma frequency, $\left.\omega_{\mathrm{p}}{ }^{*}\right)$ down to the infrared. Here, it is shown that epitaxial strain and carrier concentration allow fine tuning of optical properties $\left(\omega_{\mathrm{p}}^{*}\right)$ of $\mathrm{SrVO}_{3}$ films by modulating $\mathrm{m}^{*}$ due to strain-induced selective symmetry breaking of $3 \mathrm{~d}-\mathrm{t}_{2 \mathrm{~g}}(\mathrm{xy}, \mathrm{yz}, \mathrm{xz})$ orbitals. Interestingly, the DC electrical properties can be varied by a large extent depending on growth conditions whereas the optical transparency window in the visible is basically preserved. These observations suggest that the harsh conditions required to grow optimal $\mathrm{SrVO}_{3}$ films may not be a bottleneck for their future application.

\section{Introduction}

Transition metal oxides are among the most studied materials due to the tremendous variety of properties they may display, such as superconductivity, metal-insulator transition or multiferroicity. ${ }^{[1]}$ High electrical conductivity and carrier mobility are critical requirements for the implementation of these materials in some advanced electronic components. ${ }^{[2]}$ However, the room-temperature carrier mobility $(\mu)$ of the much explored $\mathrm{SrTiO}_{3}$ n-type semiconductor, for instance, in which the conduction band derives from rather localized $3 \mathrm{~d}$ orbitals, is only $\approx 10 \mathrm{~cm}^{2} \mathrm{~V}^{-1} \mathrm{~s}^{-1}$. Similarly, the much larger carrier mobility discovered in $\mathrm{LaAlO}_{3} / \mathrm{SrTiO}_{3}$ and related interfaces, is limited to low temperatures. ${ }^{[3]}$ In a different approach, focus was directed towards semiconductors made out of late transition metals, such as $\mathrm{BaSnO}_{3}$ (BSO). In BSO, the relatively broad conduction band derived from $\mathrm{Sn}-5 \mathrm{~s}$ orbitals anticipates a larger carrier mobility. Indeed, this has been experimentally observed by the breakthrough report of $\mu \approx 320 \mathrm{~cm}^{2} \mathrm{~V}^{-1} \mathrm{~s}^{-1}$ in La-doped $\mathrm{BaSnO}_{3}$ (La-BSO) single crystals. ${ }^{[4]}$ Unfortunately, in La-BSO thin films the carrier mobility was found to be substantially reduced, ${ }^{[5,6]}$ although molecular beam epitaxy (MBE) growth technique and proper substrate selection ${ }^{[7]}$ have allowed to partially recover $\left(\approx 150 \mathrm{~cm}^{2} \mathrm{~V}^{-1} \mathrm{~s}^{-1}\right)$ the single-crystal 
mobility value. Anyhow, in these semiconducting materials, metallicity is obtained via doping and, in consequence, optimal conductivity can only be achieved via the subtle balance between the doping concentration and mobility, that typically vary in the opposite direction upon doping. ${ }^{[8]}$ In an essentially different approach, the attention has recently shifted towards intrinsically conducting oxides made of early transition metal elements such as: $\mathrm{V}, \mathrm{Nb}, \mathrm{Mo}$, etc. In the case of $\mathrm{SrVO}_{3}(\mathrm{SVO})$, for example, the partial occupation of the $\mathrm{V}-3 \mathrm{~d}$ band $\left(3 \mathrm{~d}^{1}\right.$ electronic configuration) is responsible of the metallic conductivity. In this material the valence band is made out of $\mathrm{O}-2 \mathrm{p}$ orbital which lies well below the $3 \mathrm{~d}$ band and a large optical band gap exists $(\approx 3 \mathrm{eV})$ which guarantees no photon absorption in the visible range. ${ }^{[9]}$ Moreover, the intrinsically narrow V-3d band should lead to relatively strong electronelectron correlations and consequently to an enhanced effective mass. The large carrier concentration $(\approx 1$ electron per unit cell) and large effective mass combine to produce a plasma frequency $\left(\omega_{\mathrm{p}}^{*}\right)$ near infrared and thus SVO is found to be metallic and transparent in the visible optical range. ${ }^{[9]}$ In recent years, SVO thin films have been grown by a number of techniques, including hybrid-MBE ${ }^{[9-12]}$ and pulsed laser deposition (PLD) ${ }^{[13-22]}$, and roomtemperature resistivity (respectively mobility) values have been found to be ranging from 200 $\mu \Omega \mathrm{cm}$ (resp. $0.8 \mathrm{~cm}^{2} \mathrm{~V}^{-1} \mathrm{~s}^{-1}$ ) to $28 \mu \Omega \mathrm{cm}\left(\right.$ resp. $10 \mathrm{~cm}^{2} \mathrm{~V}^{-1} \mathrm{~s}^{-1}$ ) in best films.

The combination of large electrical conductivity and optical transparency in the visible range is a bonus of strong interest in photovoltaics, plasmonics ${ }^{[23,24]}$ or information technologies and not surprisingly, correlated transparent oxides are attracting much attention. A question then arises: which are the factors limiting the carrier mobility and the transparency window of SVO thin films?

In epitaxial films several factors may come into play. First, epitaxial strain may break the degeneracy of the cubic environment of the metallic vanadium cation in SVO by splitting the V-3d- $\mathrm{t}_{2 \mathrm{~g}}$ triplet (Figure 1a). As a consequence, the conduction band width (W) and the 
electron orbital occupancy of the $t_{2 g}$ orbitals can be modified, affecting electron-electron correlations $^{[25]}$ and carrier mobility. Interestingly, it was reported that chemical pressure in $\mathrm{Ca}_{1-\mathrm{x}} \mathrm{Sr}_{\mathrm{x}} \mathrm{VO}_{3}$ induces a gradual deformation of the unit cell that tunes $\mathrm{W}$ and, subsequently, the electronic ${ }^{[26]}$ and optical ${ }^{[27]}$ properties. It is understood that shorter V-O-V bonds in $\mathrm{CaVO}_{3}$ compared to those of $\mathrm{SrVO}_{3}$ are established via bending of the $\mathrm{V}-\mathrm{O}-\mathrm{V}$ bond angle away from the $\theta=180^{\circ}$ observed in SVO. This bending reduces $\mathrm{W}$ and increases the electronelectron correlations with a subsequent increase of the carrier effective mass $\left(\mathrm{m}^{*}\right)$ and reduction of the plasma frequency. However, a recent report on SVO films epitaxially grown on substrates having different structural mismatch suggests that the electrical and optical properties of the SVO films vary depending on the substrate used although the variation does not correlate with epitaxial strain. ${ }^{[28]}$ Intriguingly, the reported plasma frequency $\omega_{\mathrm{p}} *\left(\hbar \omega_{\mathrm{p}} * \approx\right.$ $2.3 \mathrm{eV})^{[28]}$ is substantially larger than that early measured in bulk and epitaxial films $(\approx 1.3$ eV). ${ }^{[9,26,29]}$ Second, during growth, point defects, associated to non-stoichiometry or others, may arise compromising $\mu$ (Figure 1b). ${ }^{[12]}$ Third, structural mismatch between film and substrate produces a mechanical stress that can induce plastic deformations by strain relaxation in the film structure, which should also affect $\mu$ (Figure 1c). These latter effects have been found to be of relevance in La-BSO films and related materials. ${ }^{[30]}$ Finally, in ultrathin SVO films, only few unit cells thick, quantum confinement may also give rise to selective orbital occupancy within the $\mathrm{t}_{2 \mathrm{~g}}$ manifold, ${ }^{[31]}$ but this range of film thicknesses is beyond the present scope.

In this work, we aim at disentangling the role of these potential contributions to the electronic and optical properties of SVO thin films grown by PLD. Due to their outmost relevance for electro-optic applications, we shall focus on carrier density and mobility, as well as the plasma frequency, and their dependence on structural mismatch with the substrates and 
growth conditions, namely the oxygen partial pressure and temperature. It turns out that, under optimized growth conditions and for SVO films tens of nanometer thick, the structural mismatch governs the carrier mobility and film conductivity, both decreasing in films grown on mismatched substrates. It is observed that SVO films grown on substrates imposing a tensile strain are epitaxially stressed but films grown on substrates imposing a compressive strain of similar magnitude, are relaxed. In any event, misfit-related defects are observed by electron microscopy, blurring to some extent genuine epitaxial strain effects on electronic transport properties. Interestingly, we have observed that this detrimental effect can however be partially mitigated by increasing the carrier concentration favoring the appearance of charge-screened defects (Figure 1c). Spectroscopic ellipsometry and infrared reflectivity have been used to derive the plasma frequency $\omega_{\mathrm{p}} *$ and its dependence on structural mismatch and growth conditions. Two conclusions emerge. First, for all films, $\left.\hbar \omega_{\mathrm{p}} * \approx 1.2-1.3 \mathrm{eV}\right)$ is found to scale with the carrier concentration. Second, the above observation suggests that electron bandwidth and orbital occupancy of the $t_{2 g}$ states are sensitive to strain (Figure 1a), as we have confirmed by X-ray absorption and X-ray linear dichroism measurements at vanadium $\mathrm{L}_{2,3}$ edges. It thus follows that carrier mobility in SVO films can be modulated by suitable choice of substrate and growth conditions while preserving a large transparency and the cutoff plasma energy below the visible spectrum.

\section{Experimental Results}

\subsection{Growth Window}

We first determined the growth window of SVO thin films in the $\mathrm{PO}_{2}\left(4 \times 10^{-7}\right.$ mbar to $1 \times 10^{-4}$ mbar) and $\mathrm{T}\left(700^{\circ} \mathrm{C}\right.$ to $\left.800^{\circ} \mathrm{C}\right)$ ranges on (001) STO and (001) LSAT. The room-temperature resistivity $(\rho)$ and crystalline phases (pure $\mathrm{SrVO}_{3}$ films or coexistence of $\mathrm{SrVO}_{3}$ and $\mathrm{Sr}_{3} \mathrm{~V}_{2} \mathrm{O}_{8}$ phases were obtained) are summarized in the $\left(\rho, \mathrm{T}, \mathrm{PO}_{2}\right)$ diagram shown in Figure 2, where 
we include the room-temperature resistivity of the films on LSAT and STO $v s$ the $\left(\mathrm{T}, \mathrm{PO}_{2}\right)$ growth parameters. It can be appreciated that the main trends are common to both LSAT and STO substrates.

Data in Figure 2 show that single-phase and highly conducting SVO films are obtained at the lowest oxygen partial pressure $\left(\mathrm{PO}_{2} \approx 4 \times 10^{-7}\right.$ mbar which corresponds to the base pressure of the chamber) whereas when increasing $\mathrm{PO}_{2}$, films are multi-phase $\left(\mathrm{SrVO}_{3}, \mathrm{Sr}_{3} \mathrm{~V}_{2} \mathrm{O}_{8}\right)$ and less conducting. Films grown at $\mathrm{PO}_{2}=4 \times 10^{-7}$ mbar show only the (001) reflections of the SVO perovskite indicating that the films are (001) textured, without traces (within the experimental sensitivity) of spurious phases. It can be appreciated in Figure 2 that films grown around 750$800{ }^{\circ} \mathrm{C}$ present the lower resistivity. The roughness of the films increases with $\mathrm{PO}_{2}$. AFM images are shown in Supporting Information S1. Illustrative values of resistivity and roughness (rms) of films grown at $750^{\circ} \mathrm{C}$, are: $\rho \approx 82 \mu \Omega \mathrm{cm}$ and $\mathrm{rms} \approx 0.41 \mathrm{~nm}$ for the film deposited on LSAT and $\rho \approx 200 \mu \Omega \mathrm{cm}$ and $\mathrm{rms} \approx 0.52 \mathrm{~nm}$ for the film deposited on STO. The resistivity values compare well with those early reported for thin films grown either by PLD $(30-200 \mu \Omega \mathrm{cm})^{[14,15,17,29]}$ or hybrid-MBE $(\approx 30-40 \mu \Omega \mathrm{cm})^{[9,12]}$ as we shall analyze in detail below.

Interestingly, in Figure 2 it can be appreciated that in all cases, the resistivity values of SVO//LSAT films are significantly smaller than those of the SVO//STO films. As the structural mismatch $\mathrm{f}(\mathrm{STO})$ is larger $(+1.59 \%)$ than $\mathrm{f}(\mathrm{LSAT})(+0.65 \%)$, it may be hypothesized that $\mathrm{f}$, and the associated elastic or plastic responses of the SVO lattice, may play an important role on carrier transport in SVO films. In the following, we shall use the optimal growth conditions determined above $\left(\mathrm{PO}_{2} \approx 4 \times 10^{-7} \mathrm{mbar}, \mathrm{T}=750^{\circ} \mathrm{C}\right)$ to explore in a systematic manner the role of structural mismatch between SVO and the substrates (STO, LSAT, NGO and LAO), on the electrical, optical, spectroscopic properties and the 
microstructure of the films. Surface morphology analysis (topographic images are in Supporting Information S2) shows that, irrespectively of the substrate, films grown under these conditions are remarkably flat, with $\mathrm{rms} \approx 0.52 \mathrm{~nm}(\mathrm{STO}), 0.41 \mathrm{~nm}(\mathrm{LSAT}), 0.46 \mathrm{~nm}$ (NGO) and $0.48 \mathrm{~nm}$ (LAO). In agreement with earlier findings, ${ }^{[15]}$ when increasing $\mathrm{PO}_{2}, \mathrm{SVO}$ films display the gradual formation of outgrowths at their surface, related to the formation of spurious $\mathrm{Sr}_{3} \mathrm{~V}_{2} \mathrm{O}_{8}$ phase as evidenced by XRD data shown below.

\subsection{Structural Properties}

The XRD $\theta-2 \theta$ scans of films deposited under the optimal conditions $\left(750^{\circ} \mathrm{C}, 4 \times 10^{-7} \mathrm{mbar}\right)$ on all substrates, zoomed around the (002) reflection of the corresponding substrates, are shown in Figure 3a.

We first focus on the SVO film on STO which has the largest tensile structural mismatch $(\mathrm{f}(\mathrm{STO})=+1.59 \%)$. The $(002)$ reflection of the SVO film (Figure 3a (green curve)), is located at the right of the (002) STO reflection, and attentive inspection reveals Laue fringes (Supporting Information S3). Interestingly, the (002) SVO reflection occurs at lower angle than that expected for bulk SVO (indicated by a vertical dashed line in Figure 3a), implying an expanded c-axis (c(SVO//STO) $\approx 3.873 \AA)$. Therefore, the observed expansion of c-axis is not due to the stress (tensile) imposed by the substrate but is likely related to growth-induced defects, including non-stoichiometry, as commonly found in SVO films. ${ }^{[11,12,16,17]}$ The reciprocal space maps (Supporting Information S4) show that the (002) SVO and (002) STO reflections are well aligned along [100] indicating that the in-plane cell parameter of SVO and STO are closely coincident and thus SVO is in-plane strained $(\mathrm{a} \approx 3.905 \AA)$. Therefore, the $\mathrm{VO}_{6}$ coordination polyhedron in SVO//STO has c/a $<1$.

In the opposite limit, for SVO//LAO films where a large compressive mismatch exists $(f(L A O)=-1.37 \%)$, two broad $(002)$ SVO reflections can be observed in the XRD pattern 
(top violet curve). One occurring at $(2 \theta)_{1} \approx 46.94^{\circ}$, indicates an out-of-plane cell parameter $\mathrm{c}_{1}(\mathrm{SVO} / / \mathrm{LAO}) \approx 3.868 \AA$ and the other, located at $(2 \theta)_{2} \approx 46.3^{\circ}$, indicates a larger c-axis: $\mathrm{c}_{2}(\mathrm{SVO} / / \mathrm{LAO}) \approx 3.919 \AA$ A. These reflections occur at smaller $2 \theta$ angles than bulk (002) SVO (vertical dashed line); hence both peaks correspond to larger c-axis than bulk SVO. Although this behavior could be expected if the negative mismatch of the LAO substrate on the film would induce a compressive epitaxial strain on the basal plane of SVO, the reciprocal space maps (Supporting Information S4) indicate that the cell parameters of SVO are not clamped to those of the substrate but relaxed. Therefore, for SVO//LAO, the $\mathrm{VO}_{6}$ coordination polyhedron has an enhanced tetragonality c/a $>1$.

The X-ray reflections of SVO films on LSAT and NGO are hardly discernible from those of the substrate due to the close structural matching and a noticeable SVO line-broadening that, in accordance with topographic images (Supporting Information S2), can be attributed to the limited size (grain size $<50 \mathrm{~nm}$ ) of the coherently-diffracting volume, shrank by the presence of strain-induced defects (see Section 2.4). From the $\theta-2 \theta$ and reciprocal space maps (Supporting Information S4) we determine that the corresponding c-axis parameters are $\mathrm{c}(\mathrm{SVO} / / \mathrm{LSAT}) \approx 3.87 \AA$ and $\mathrm{c}(\mathrm{SVO} / / \mathrm{NGO}) \approx 3.86 \AA$, while the in-plane parameters coincide with those of the corresponding substrates. Consistently with the results of the SVO//STO above, SVO films on NGO and LSAT films are epitaxially strained. Therefore, within the experimental resolution, for $\mathrm{SVO} / / \mathrm{NGO}$ and $\mathrm{SVO} / / \mathrm{LSAT} \mathrm{c} / \mathrm{a} \approx 1$.

In Figure 3b, we plot the c-axis parameters of all films. We also include the predicted cell parameter of epitaxial strained SVO films on the different substrates, calculated using the Poisson equation and assuming volume conservation. ${ }^{[12]}$ It is clear that the c-axis values of the films, excluding $\mathrm{c}_{1}(\mathrm{SVO} / / \mathrm{LAO})$, are larger than that expected if a pure elastic compressive or tensile strain were acting on the SVO film. As mentioned above, a unit cell expansion is commonly observed in SVO films and attributed non-stoichiometric defects related to the 
extremely low $\mathrm{PO}_{2}$ used during growth. ${ }^{[11,12,16,17]}$ Recently, it has been reported that using a non-reactive gas in the PLD growth process, this effect can be mitigated ${ }^{[29]}$ probably as a $^{2}$ result of changing the $\mathrm{Sr} / \mathrm{V}$ ratio or the oxidation state of species in the plume. ${ }^{[13]}$

The symmetric X-ray diffraction $\theta-2 \theta$ scans do not reveal the presence of spurious phases in none of films grown at low pressure $\left(\mathrm{PO}_{2}=4 \times 10^{-7} \mathrm{mbar}\right)$. However, as the corresponding reflections may not be visible in symmetric scans, we collected 2D maps of the reciprocal space along $2 \theta$ and $\chi$ angles. In Figure $3 \mathrm{c}$ (top panel) we show the $2 \theta-\chi$ frame for SVO//STO samples. The intense (001) and (002) substrate reflections are well visible. The superimposed (001) and (002) SVO reflections are not distinguishable from those of the substrate, which is in agreement with the $\theta-2 \theta$ scans (Figure 3a) and the lower resolution of the 2D detector. Importantly, no other reflections that could suggest the presence of spurious phases are visible in these films. We then conclude that films grown at the lowest pressure $\left(\mathrm{PO}_{2}=4 \times 10^{-7} \mathrm{mbar}\right)$ are single phase, as summarized in Figure 2. However, this is not the case for films grown at higher $\mathrm{PO}_{2}$. In Figure 3c (bottom panel) we show a $2 \theta-\chi$ frame of the SVO//STO sample grown at $\mathrm{PO}_{2}=2 \times 10^{-5}$ mbar. In this map, additional spots that correspond to the (205) $\mathrm{Sr}_{3} \mathrm{~V}_{2} \mathrm{O}_{8}$ reflections are visible. Consistently, the AFM images of this SVO//STO sample (Supporting Information S1) show outgrowths which are associated to the $\mathrm{Sr}_{3} \mathrm{~V}_{2} \mathrm{O}_{8}$ phase identified in the 2D maps. ${ }^{[15]}$ Similar results are observed in films grown at high $\mathrm{PO}_{2}$ on LSAT substrates.

\subsection{Electrical Transport Properties}

We turn now to the electrical transport properties of the films with the focus on its dependence on structural mismatch and the $\mathrm{PO}_{2}$ used during growth. In Figure 4a we show the room-temperature resistivity $\rho(300 \mathrm{~K})$ of the SVO films grown on different substrates at 
$\mathrm{PO}_{2}=4 \times 10^{-7}$ mbar (square symbols) and $\mathrm{PO}_{2}=2 \times 10^{-5}$ mbar (circle symbols). The resistivity is minimal for the films grown on best matched substrates (LSAT and NGO) and, for a given mismatch, the resistivity decreases when reducing $\mathrm{PO}_{2}$. Accordingly, $\rho(300 \mathrm{~K})$ reaches its smallest value (at $\mathrm{PO}_{2}=4 \times 10^{-7} \mathrm{mbar}$ ) for $\mathrm{SVO} / / \mathrm{NGO}$ films, where $\mathrm{f}(\mathrm{SVO} / / \mathrm{NGO})=+0.52 \%$. The resistivity value $\rho(300 \mathrm{~K}, \mathrm{NGO}) \approx 85 \mu \Omega \mathrm{cm}$ is comparable to the state-of-the-art resistivity values of films of similar thickness grown, under similar conditions, by PLD $(\approx 35$ $90 \mu \Omega \mathrm{cm})^{[13,15,17,29]}$ and only 3 times larger than that of films grown by hybrid-MBE $(\approx 30-40$ $\mu \Omega \mathrm{cm}) \cdot{ }^{[9,12]}$ As mentioned above, the use of a non-reactive gas during PLD growth allows to reduce films non-stoichiometry and to lower their resistivity $\rho(300 \mathrm{~K})$ down to $\approx 31 \mu \Omega \mathrm{cm}$; ${ }^{[29]}$ more importantly here, the overall trend of $\rho(300 \mathrm{~K})$ with the lattice mismatch is preserved. ${ }^{[29]}$ The same trend of $\rho(300 \mathrm{~K})$ with the lattice mismatch is also observed in the series of films grown at $\mathrm{PO}_{2}=2 \times 10^{-5}$ mbar (circles). Therefore, data in Figure 4a indicates an important role of the film-substrate mismatch on the resistivity of SVO films.

The carrier concentration (n) and mobility $(\mu)$ values, extracted from resistivity and Hall effect measurements, of films grown at the lowest $\mathrm{PO}_{2}\left(4 \times 10^{-7} \mathrm{mbar}\right)$ on different substrates, are shown in Figure $4 \mathrm{~b}$ (diamonds and squares, respectively). We first note that the carrier concentration is almost constant $\left(\mathrm{n} \approx 2.1 \times 10^{22} \mathrm{~cm}^{-3}\right)$ for films on LSAT, NGO and LAO. This value is close to, but slightly larger than the value expected for stoichiometric SVO with $\mathrm{V}^{4+}$ $\left(3 \mathrm{~d}^{1}\right)\left(\approx 1.76 \times 10^{22} \mathrm{~cm}^{-3}\right)$. A perceptible larger $16 \%$ carrier concentration is observed for films grown on STO. We note that if the STO substrate would have opened a conducting parallel channel in the SVO//STO sample grown at the lowest pressure, then the measured carrier density would have been smaller but not larger. Therefore, a plausible reason is that the tensile strain imposed by the STO substrate favors a higher concentration of oxygen vacancies, that can provide additional carriers while reducing mobility. ${ }^{[32]}$ 
The carrier mobility reaches its largest value $\left(\approx 3.3 \mathrm{~cm}^{2} \mathrm{~V}^{-1} \mathrm{~s}^{-1}\right)$ for SVO grown on the best matching NGO substrate, being marginally smaller in films on LSAT and LAO. A more pronounced reduction $\left(\approx 1.2 \mathrm{~cm}^{2} \mathrm{~V}^{-1} \mathrm{~s}^{-1}\right)$ is observed in SVO//STO, consistent with an increased strain-related non-stoichiometry, as indicated above. As shown by Mirjolet et al., ${ }^{[29]}$ the use of a non-reactive gas during the PLD growth allows to increase the mobility up to above $8 \mathrm{~cm}^{2} \mathrm{~V}^{-1} \mathrm{~s}^{-1}$, approaching the record values obtained in hybrid-MBE films $\left(\approx 10 \mathrm{~cm}^{2} \mathrm{~V}^{-}\right.$ $\left.{ }^{1} \mathrm{~S}^{-1}\right) \cdot{ }^{[9]}$

The temperature dependence of the resistivity $\rho(T)$ and the residual resistivity ratio $(R R R=$ $\rho(300 \mathrm{~K}) / \rho(5 \mathrm{~K}))$ of SVO films are also significantly different depending on the substrate used, as shown in Figure 4c and Figure 4d, respectively. It can be appreciated that the $\mathrm{SVO} / \mathrm{NGO}$ film displays the largest $\mathrm{RRR}(\mathrm{RRR} \approx 2.1)$ (Figure $4 \mathrm{~d})$ and RRR gradually decreases in films on substrates having larger lattice mismatch. For instance, RRR $\approx 1.4$ for SVO//STO. Accordingly, the maximal RRR (minimal residual resistivity) is also obtained in films grown on well-matched substrates (Figure 4d). We note in passing that the largest RRR values reported for SVO films grown by $\mathrm{PLD}$ under standard $\mathrm{PO}_{2}$ atmosphere were of only $\approx$ $1.7 \cdot[15,17]$

Overall, all data in Figure 4 strongly suggest that resistivity, carrier density and mobility of SVO films grown under optimized conditions are primarily determined by the structural mismatch with substrates, maybe exacerbated by mismatch-controlled non-stoichiometry. It is known that defects in solids, affecting carrier mobility, can be screened by carriers and therefore, the mobility is affected by the carrier density in an unconventional manner. ${ }^{[33]}$ To explore this effect, we focus on SVO//LSAT and SVO//STO films as illustrative examples of well and poor matched substrates, respectively, and analyze the relationship between carrier density and mobility of SVO films. In Figure 5 we plot $\mu \nu s$ for SVO//LSAT (squares) and SVO//STO (circles) films grown at the same temperature $\left(750^{\circ} \mathrm{C}\right)$ and varying $\mathrm{PO}_{2}$. Data in 
Figure 5 reveal important trends. First, at any $\mathrm{PO}_{2}$ the carrier mobility is larger for films grown on LSAT than on STO, thus confirming data in Figure 4. Next, it is observed that for films on both substrates, an enhancement of mobility goes in parallel with an increase of carrier concentration $\left(\mu \approx \mathrm{n}^{\mathrm{b}}, \mathrm{b}>0\right)$. The relevance of this observation is better appreciated by noticing that in conventional doped semiconductors, the opposite behavior is typically observed. Indeed, it is commonly found that $\mu$ decreases with increasing doping due to the enhanced scattering of carriers with dopant atoms. The reverse trend observed here bears some resemblance with properties of some strained semiconductors, such as $\mathrm{BaSnO}_{3}$, where a similar $\mu \approx \mathrm{n}^{\mathrm{b}}$ with $\mathrm{b}>0$ have been reported. ${ }^{[6,30]}$ It has been argued that this unusual increase of mobility when increasing the carrier density is due to the enhanced screening of extended defects, such as dislocations, by the carriers (Figure 1c). ${ }^{[33]}$ The confined and directional character of the relevant $3 \mathrm{~d}$ orbitals forming the conduction band of SVO advances a strong sensitivity of carrier mobility to extended structural defects. Therefore, it may not be a surprise that the carrier-induced screening can efficiently increase mobility.

\subsection{Microstructure}

The observations above suggest that extended defects associated to structural mismatch play a major role on charge scattering and trapping. To get insight on this, the microstructure of the films grown at $\mathrm{PO}_{2}=4 \times 10^{-7}$ mbar and $\mathrm{T}=750^{\circ} \mathrm{C}$ were studied by TEM. Specimens from the SVO films grown on LAO, NGO and STO were prepared in cross section geometry by FIB lift-out technique. Figure 6(a-c) (main panels) shows bright field images of SVO//LAO, SVO//NGO and SVO//STO, respectively. It can be appreciated that all films present a sharp interface (see horizontal solid lines) with the substrate, homogeneous thickness of $\approx 70 \mathrm{~nm}$ and a free surface with a roughness at the nanometer level. From these bright field images acquired in zone axis conditions, it can be seen that the films present an incoherent contrast, 
with obvious dissimilarities among them. In order to assess the density and nature of the crystal defects that may be responsible for the observed contrast, the crystals were oriented in two-beam conditions by tilting the specimen in the TEM. In opposition to zone axis condition, in which the crystal is oriented so that the Ewald sphere is tangent to a plane of nodes of the reciprocal lattice of the crystal, in two-beam conditions the crystal is oriented in such a way that only a family of reflections is in Bragg condition (the second beam being the direct beam). By acquiring bright field images in this condition, defects in the direction given by the intersection of the Ewald sphere with the reciprocal lattice of the crystal are strongly highlighted.

The bright field images acquired in two beam conditions described above, are shown in the insets of Figure 6(a-c). Different two beam conditions were set for all the SVO films: one corresponding to the family of planes stacked in the growth direction and the other in the perpendicular direction. In Figure 6a (inset) we show an image collected for the SVO film grown on the LAO substrate, which we remind here is subjected to a compressive stress. The image reveals a microstructure of defects consisting on planar defects oriented parallel to the substrate (compressive stress, LAO) (Figure 6a (inset)) or perpendicular to the substrate (tensile stress, STO) (Figure 6c (inset)). In contrast, planar defects oriented perpendicular to the substrate can be observed in SVO grown on substrates imposing a tensile stress (STO) (Figure 6c (inset)). The presence of oriented planar defects in thin films grown on mismatched substrates, has been reported in other perovskite thin films, such as $\mathrm{La}_{0.5} \mathrm{Sr}_{0.5} \mathrm{CoO}_{3-\mathrm{x}}{ }^{\left[{ }^{[3-36]}\right.}$ or $\mathrm{LaNiO}_{3-\mathrm{x}}{ }^{[37]}$ to name a few, and it is believed to be a signature of defects ordering (oxygen vacancies) to release epitaxial stress. More precisely, high resolution electron microscopy images of $\mathrm{La}_{0.5} \mathrm{Sr}_{0.5} \mathrm{CoO}_{3-\mathrm{x}}$ films grown on LAO and STO substrates, imposing -as in the present case- compressive and tensile stress respectively, clearly revealed a similar arrangement of planar defects. ${ }^{[36]}$ In the present case, it cannot be excluded that planar defects 
could be related to the presence of $\mathrm{Sr}_{3} \mathrm{~V}_{2} \mathrm{O}_{8}$, as identified in some XRD data. However, the observation that in SVO film grown on NGO substrate (Figure 6b (inset)), with a very small lattice mismatch, a much smaller density of defects was observed in both crystal orientations seems to favor the former scenario. The presence of these extended planar defects should impact the electronic transport. Indeed, for SVO//STO where the array of defects is perpendicular to the interface, the carrier mobility should be much obstructed than in SVO//LAO where these defects lay parallel to the interface. This is indeed, the trend observed in Figure 4b.

\subsection{Optical Properties}

Spectroscopic ellipsometry (SE) measurements were performed on SVO//(STO, LSAT, NGO and LAO) films grown at $\mathrm{PO}_{2}=4 \times 10^{-7} \mathrm{mbar}$ and at $\mathrm{PO}_{2}=2 \times 10^{-5}$ mbar to elucidate their optical properties and determine their plasma frequency $\omega_{\mathrm{p}}{ }^{*}$. The real and imaginary parts of the dielectric constants $\left(\varepsilon=\varepsilon_{1}+i \varepsilon_{2}\right)$ and optical coefficients $(\tilde{n}=n+i \kappa)$ were extracted from the SE data (see experimental section). Figure 7a,b shows the spectral dependence of $\varepsilon_{1}$ and $\varepsilon_{2}$ of some illustrative films. The shape of both components of the complex permittivity $\varepsilon$ are in good agreement with earlier reports. ${ }^{[9]}$ From $\varepsilon_{1}(\omega)$ the screened plasma frequency $\omega_{\mathrm{p}} *$ can be determined by using the condition $\varepsilon_{1}\left(\omega_{\mathrm{p}}^{*}\right)=0$. Two salient features emerge from data in Figure 7a. First, $\omega_{\mathrm{p}} *$ increases when reducing $\mathrm{PO}_{2}$ during growth and second, for a given growth $\mathrm{PO}_{2}, \omega_{\mathrm{p}}{ }^{*}$ is reduced in $\mathrm{SVO} / \mathrm{STO}$ compared to SVO//LSAT. These trends can be better visualized in Figure $7 \mathrm{c}$ where we plot $\mathrm{E}_{\omega_{\mathrm{p}} * v s} \mathrm{f}$ values of these films. As seen, all $\hbar \omega_{\mathrm{p}} *$ values are around 1.2-1.3 eV, which is agreement with earlier reports for SVO//LSAT. ${ }^{\left[{ }^{2,2]}\right.}$ To get a further insight on the implications of these observations, we recall that ${ }^{[26]}$

$$
\left(E_{\omega p^{*}}\right)^{2}=\frac{\hbar^{2} e^{2}}{\varepsilon_{0} \varepsilon_{\infty}} \frac{1}{m^{*}} \times n
$$


where $\varepsilon_{\infty}$ is the high frequency relative permittivity of the medium, $\mathrm{n}$ is the free carrier density, $\mathrm{m}^{*}$ is the free carrier effective mass, and $\hbar$, e and $\varepsilon_{0}$ stand for the reduced Planck constant, the electron charge and the free space permittivity, respectively. In Figure $7 \mathrm{~d}$ we plot $\mathrm{E}_{\omega \mathrm{p}^{*}}{ }^{2} v s \mathrm{n}$ (where $\mathrm{n}$ is the carrier density determined from Hall effect, see Figure 4). This plot allows to conclude that the plasma energy increases when reducing $\mathrm{PO}_{2}$ because the carrier density increases. Moreover, the smaller slope of the $\mathrm{E}_{\omega \mathrm{p}^{*}}{ }^{2}(\mathrm{n})$ plot observed in SVO//STO anticipates a larger effective mass of carriers in comparison to SVO//LSAT. Using $\varepsilon_{\infty}=4$, as determined in bulk SVO, ${ }^{[26]}$ the effective mass $\mathrm{m}^{*}$ can be computed from data in Figure 7 d. For SVO//LSAT, we obtain $\mathrm{m}^{*}(\mathrm{LSAT}) \approx 4$ irrespectively on the $\mathrm{PO}_{2}$. For $\mathrm{SVO} / / \mathrm{STO}$ we obtain a significant larger mass $\mathrm{m}^{*}(\mathrm{STO}) \approx 5$, which represents a $25 \%$ enhancement. It is worth to recall that in $\mathrm{Sr}_{1-\mathrm{x}} \mathrm{Ca}_{\mathrm{x}} \mathrm{VO}_{3}$ bulk materials, $\mathrm{m}^{*}$ was found to increase from 3.3 to about 4 when increasing $x$ due to internal chemical pressure. ${ }^{[26]}$

The complex refractive index of the film and substrate extracted from the ellipsometry measurements were used to calculate the sample reflectivity $R_{\text {calc }}(\omega)$, assuming a simple model consisting of a substrate and a SVO film, to compare it with experimental data. FTIR measurements have been used to determine $\mathrm{R}_{\exp }(\omega)$ at normal incidence. The experimental $\mathrm{R}_{\exp }(\omega)$ and $\mathrm{R}_{\text {calc }}(\omega)$ data for some illustrative films are shown in Supporting Information S5. Data reveal that $\mathrm{R}(\omega)$ displays a minimum at around $600 \mathrm{~nm}(\approx 2 \mathrm{eV})$. This observation is in agreement with results from Boileau et al. ${ }^{[28]}$ although we emphasize that the minimum of $\mathrm{R}(\omega)$ cannot be taken as a measure of $\omega_{\mathrm{p}}{ }^{*}$, due to the substrate contribution to the reflectivity measurements. It is of the highest interest to notice that all samples, irrespectively of their DC conductivity, display a remarkably small $\varepsilon_{2}(\omega)$ in the visible range (Figure $7 b$ ), which is a signature of small absorption. It thus follows that, within the explored growth conditions 
range, although electrical conductivity and plasma frequency can be modulated, the optical absorption remains substantially unperturbed.

\subsection{X-Ray Absorption and Orbital Occupancy}

In section 3.5 we have shown that the effective mass of carriers in SVO films on STO is larger than that of SVO films on LAO, LSAT and NGO substrates. As films on different substrates appear to be under different strain state, a natural question arises: Which is the role of strain on orbital occupancy and ultimately on bandwidth broadening? Indeed, it is well known that substrate-induced stress on epitaxial films of oxides breaks the orbital degeneracy and promotes a selective electron occupancy in well-defined orbitals, that affects transport properties. ${ }^{[38]}$ The X-ray absorption (XAS) at metal $\mathrm{L}_{2,3}$ edges in epitaxial metal oxide films, is sensitive to the relative orientation of the polarization direction of the incoming X-ray beam with respect to the film surface. This gives rise to an X-ray linear dichroism (XLD), defined as $\mathrm{XLD}=\mathrm{I}\left(E_{\mathrm{ab}}\right)-\mathrm{I}\left(E_{\mathrm{c}}\right)$, different from zero if final states with different symmetry are not equally available; ${ }^{[39]}$ where $\mathrm{I}\left(E_{\mathrm{ab}}\right)$ and $\mathrm{I}\left(E_{\mathrm{c}}\right)$ are the intensities of the absorption of light with the electric field (polarization) parallel to the film plane $\left(E_{\mathrm{ab}}\right)$ or perpendicular to it $\left(E_{\mathrm{c}}\right)$. For an epitaxial (001) SVO film, as in the present case, $E_{\mathrm{ab}}$ is probing electronic states with (xy) symmetry and $E_{\mathrm{c}}$ is probing electronic states with (xz, yz) symmetry. In the particular case of $\mathrm{V}-3 \mathrm{~d}^{1}$, if the electron occupancy at the (xy) and (xz, yz) orbitals is not identical, a non-zero XLD should be apparent. XLD will be different depending if the lower lying state is (xy) or $\left(\mathrm{xz}, \mathrm{yz}\right.$ ), that is depending on the sign of the energy difference $\Delta \mathrm{t}_{2 \mathrm{~g}}=\mathrm{E}_{\mathrm{xz}, \mathrm{yz}}-\mathrm{E}_{\mathrm{xy}}$ where $\mathrm{E}_{\mathrm{xy}}$ and $\mathrm{E}_{\mathrm{xz}, \mathrm{yz}}$ are the corresponding energies. Notice that $\Delta \mathrm{t}_{2 \mathrm{~g}}>0$ has to be found in presence of an inplane expansion of the equatorial bonds in $\mathrm{VO}_{6}$ and $\Delta \mathrm{t}_{2 \mathrm{~g}}<0$ corresponds to an out-of-plane expansion of $\mathrm{O}-\mathrm{V}-\mathrm{O}$ bonds in $\mathrm{VO}_{6}$. 
In Figure 8a we show the combined V-L $\mathrm{L}_{2,3}$ and O-K XAS spectra of the SVO films grown on STO, NGO and LAO substrates, selected because they display the maximal (STO tensile, LAO compressive) and minimal (NGO) mismatch. The V-L $\mathrm{L}_{3}$ and $\mathrm{L}_{2}$ edges (at $519 \mathrm{eV}$ and 524 $\mathrm{eV}$ ) are signatures of the dipole transitions from $2 \mathrm{p}_{3 / 2}$ to $3 \mathrm{~d}$ and from $2 \mathrm{p}_{1 / 2}$ to $3 \mathrm{~d}$ orbitals. The double peak (appearing at $530 \mathrm{eV}$ ) of the $\mathrm{O}-\mathrm{K}$ pre-peak is a fingerprint for the $\mathrm{V}^{4+}$ valence state. The relative intensity of the $\mathrm{L}_{2,3}$ as well as the splitting of the O-K pre-peak are characteristic of $\mathrm{V}^{4+}$ in an octahedral coordination. ${ }^{[40,41]}$ A detailed inspection of the low energy side of the $\mathrm{L}_{3}$ peak (see Figure $8 \mathrm{~b}$ ) reveals a fine structure where three peaks can be identified. As shown by cluster calculations ${ }^{[41]}$ the appearance of these peaks is prominent in tetragonally distorted $\mathrm{VO}_{6}$ octahedra. In Figure $8 \mathrm{~b}$ it can be appreciated that these features are more prominent in SVO//STO than in SVO//NGO and SVO//LAO, and according to the previous statement, we conclude that in $\mathrm{SVO} / \mathrm{STO}$, the $\mathrm{VO}_{6}$ octahedra have a larger tetragonal distortion than in SVO//NGO and SVO//LAO.

A more direct evidence is provided by the XLD data shown in the following. In Figure 8c we present the XLD $=\mathrm{I}\left(E_{\mathrm{ab}}\right)-\mathrm{I}\left(E_{\mathrm{c}}\right)$ data of SVO//(STO, NGO, LAO) films. To appreciate the implications of the data in Figure 8c, we remind here that theoretical cluster calculations ${ }^{[41]}$ of XLD for $\Delta \mathrm{t}_{2 \mathrm{~g}}>0$ and $\Delta \mathrm{t}_{2 \mathrm{~g}}<0$ predicts XLD spectra that are virtually opposite one another, and thus comparison with experimental data should allow to discern between $\Delta \mathrm{t}_{2 \mathrm{~g}}>0$ and $\Delta \mathrm{t}_{2 \mathrm{~g}}$ $<0$. The XLD data in Figure 8c show that for SVO films, the overall trend is similar to the above predicted $\Delta \mathrm{t}_{2 \mathrm{~g}}>0$ case. ${ }^{[41]}$ Interestingly, as clearly shown by data in Figure $8 \mathrm{c}$, the height of the most intense XLD peak, which is the most sensitive to the magnitude of $\Delta \mathrm{t}_{2 \mathrm{~g}}$ is somewhat larger in SVO//STO than in SVO//(NGO, LAO). It has also been predicted that the features appearing at the low-energy side of $\mathrm{L}_{3}$ are more sensitive to tetragonal distortion for $\Delta \mathrm{t}_{2 \mathrm{~g}}>0$ than for $\Delta \mathrm{t}_{2 \mathrm{~g}}<0$. In Figure $8 \mathrm{~b}$ it can be appreciated that these features are more visible in SVO//STO than in SVO//NGO and SVO//LAO, and according to the previous 
statement, we conclude that in $\mathrm{SVO} / / \mathrm{STO}, \Delta \mathrm{t}_{2 \mathrm{~g}}$ is positive $(>0)$ and larger than in $\mathrm{SVO} / / \mathrm{NGO}$ and SVO//LAO. Therefore, the $\mathrm{VO}_{6}$ octahedra in SVO// STO are under a larger tensile inplane stress than in $\mathrm{SVO} / / \mathrm{NGO}$ and $\mathrm{SVO} / / \mathrm{LAO}$. Although in principle $\Delta \mathrm{t}_{2 \mathrm{~g}}$ could be deduced by comparing multiplet calculations with experimental spectra, we content ourselves here by using XLD to discern between tensile or compressive strain.

Overall, from both XAS and XLD data we conclude that: (i) a tetragonal crystal field breaks the symmetry of the $\mathrm{t}_{2 \mathrm{~g}}$ manifold into (xy) and (xz, yz) states; (ii) as $\Delta \mathrm{t}_{2 \mathrm{~g}}>0$, the $\mathrm{t}_{2 \mathrm{~g}}(\mathrm{xy})$ states lay lower in energy than $t_{2 g}(x z, y z)$ and thus they have a higher electron occupancy (see Figure $8 \mathrm{~d}$ for the unstrained and strained $\mathrm{VO}_{6}$ octahedra and the $\mathrm{t}_{2 \mathrm{~g}}$-manifolds).

\section{Conclusion}

Overall, we have shown that $\mathrm{SrVO}_{3}$ films grown under different oxygen pressure and on substrates having different structural mismatch, thus imposing different (tensile or compressive) epitaxial stress, clearly show distinct transport properties. The carrier mobility is found to be the largest in SVO films grown on matching substrates and lowering when films are grown on substrates imposing a large tensile or compressive strain. Although the film conductivity, carrier density and mobility are found to depend on the growth conditions (mainly the oxygen pressure) as expected in presence of growth-induced point defects (Figure $1 \mathrm{~b}$ and Figure 4) the dependence on these parameters on substrate mismatch is fully preserved, thus suggesting that the substrate plays a major role. Interestingly, the reduction of mobility is asymmetric, being more pronounced in case of tensile stress (SVO//STO) but only marginally larger in case of compressive stress (SVO//LAO).

Using first principle calculations Sclauzero et al. ${ }^{[25]}$ have predicted that epitaxial strain on SVO has basically two effects. First, a crystal field of tetragonal symmetry breaks the degeneracy of the $t_{2 g}$ electronic triplet and, irrespectively of the sign of strain (Figure 1a), the 
electronic correlations will be reinforced and approaching SVO to an insulator Mott regime. Second, stretching the M-O bonds should have a similar effect, whereas bond shortening should have the opposite effect, that is enhancing the hopping amplitude and thus promoting a more metallic character. Therefore, for tensile strain a more insulating character of SVO is expected whereas for a compressive strain, band narrowing and hopping-amplitude enhancement cancel out their contributions and only minor changes of carrier mobility are expected. Our XAS and XLD data provide clear evidence of the strain-induced breaking of symmetry and stabilization of the xy orbitals in STO compared to other substrates (Figure 1a and Figure 8). This is consistent with the c/a $<1$ distortion of the unit cell of SVO//STO, inferred from X-ray diffraction data. Charge redistribution enhances xy orbital occupancy, narrows the electronic bandwidth and enhances the effective mass of carriers in films on STO compared to other substrates. This is precisely the result obtained from ellipsometry measurements, where $\mathrm{m} *(\mathrm{SVO} / / \mathrm{STO}) \approx 1.25 \mathrm{x} \mathrm{m*}(\mathrm{SVO} / / \mathrm{LSAT})$ (Figure 7). However, this $25 \%$ enhancement of the effective mass of carriers in SVO//STO cannot account for the observed $\approx 200 \%$ larger resistance in SVO//STO compared to SVO//LSAT (or other substrates) (Figure 4a). Therefore, other effects should play a bigger role on dc transport properties.

The TEM images have provided evidence of the existence of arrays of planar defects in SVO films grown on mismatched substrates and their orientation is compatible with the observed modification of mobility, more (less) pronounced in SVO films on STO (LAO). This is the trend observed for the resistivity and carrier mobility of SVO films grown on different substrates (Figure 4a,b). Remarkably, it is found (Figure 5) that carrier mobility increases when increasing carrier concentration. This observation favors the view that electrical conductivity and mobility in these strained films, although affected by the heavier electron mass in tensile strained films, are primarily governed by microstructural effects, namely stress-induced planar defects (Figure 1c). Therefore, one could anticipate that avoiding strain 
relaxation, maybe by using thinner SVO films, the genuine effects of band reconstruction due to epitaxial strain will become more apparent on the dc electric transport properties.

Otherwise, only optical conductivity (thus plasma frequency) is sensitive to these effects. From a practical point of view, it is remarkable that the optical transparency window remains in the visible range for all studied films irrespectively on the growth conditions and substrates, thus suggesting that the harsh conditions required to grow optimal SVO films may not be a limitation for future applications.

\section{Experimental Section}

Samples preparation: $\mathrm{SrVO}_{3}(\mathrm{SVO})$ films were grown on cubic single crystalline perovskite substrates having different cell parameters: $\mathrm{SrTiO}_{3}\left(\mathrm{STO}, 3.905 \AA\right.$ A), $\left(\mathrm{LaAlO}_{3}\right)_{0.3^{-}}$ $\left(\mathrm{Sr}_{2} \mathrm{AlTaO}_{6}\right)_{0.7}\left(\mathrm{LSAT}, 3.868 \AA\right.$ ), $\mathrm{NdGaO}_{3}\left(\mathrm{NGO}\right.$, (pseudo-cubic) $3.863 \AA$ ) and $\mathrm{LaAlO}_{3}$ (LAO, $3.791 \AA$ ). Bulk SVO is cubic with $\mathrm{a}(\mathrm{SVO})=3.842 \AA{ }^{[11,42]}$ Therefore, the structural mismatch between SVO and the substrate, defined as $\mathrm{f}=[\mathrm{as}-\mathrm{a}(\mathrm{SVO})] / \mathrm{as}$, where as is the cell parameter of the substrate is $\mathrm{f}(\mathrm{STO})=+1.59 \%, \mathrm{f}(\mathrm{LSAT})=+0.65 \%, \mathrm{f}(\mathrm{NGO})=+0.52 \%$, and $f(L A O)=-1.37 \%$. As-received (001)-oriented single crystals (cubic and pseudocubic settings) were used as substrates. Films were grown by pulsed laser deposition (PLD) (excimer laser, $248 \mathrm{~nm}$ wavelength) at a frequency of $5 \mathrm{~Hz}$, a fluence of around $2 \mathrm{~J} \mathrm{~cm}^{-2}$, and the number of laser pulses was 2000. A $\mathrm{Sr}_{2} \mathrm{~V}_{2} \mathrm{O}_{7}$ target was prepared by solid state reaction of stoichiometric amounts of $\mathrm{SrCO}_{3}$ and $\mathrm{V}_{2} \mathrm{O}_{5}$. The oxygen partial pressure $\left(\mathrm{PO}_{2}\right)$ inside the PLD chamber was varied from $\mathrm{PO}_{2}=1 \times 10^{-4}$ mbar down to the base pressure at the growth temperature, corresponding roughly to $\mathrm{PO}_{2} \approx 4 \times 10^{-7}$ mbar. The temperature of deposition was varied between $700^{\circ} \mathrm{C}$ and $800^{\circ} \mathrm{C}$. After growth, films were cooled down to room temperature by switching off the heater of the sample holder, while keeping the same pressure as used for the growth. 
Structural Characterization: $\mathrm{X}$-ray diffraction $(\mathrm{XRD})\left(\mathrm{Cu}-\mathrm{K}_{\alpha 1}\right.$ radiation) measurements were done using either a PANanalytical X'Pert MRD ( $\theta-2 \theta$ scans) or a Bruker AXS D8 Advance equipped with a GADDS $2 \mathrm{D}$ detector ( $2 \theta-\chi$ frames). Film thickness was determined by X-ray reflectivity $(\mathrm{XRR})$. Within the explored $\left(\mathrm{T}, \mathrm{PO}_{2}\right)$ range of parameters, the thickness of all films was found to be within the $70-80 \mathrm{~nm}$ range. Topographic measurements were done by using an atomic force microscope (AFM) in tapping mode. Specimens for transmission electron microscopy (TEM) observation were prepared in cross section geometry by focused ion beam (FIB) using the lift-out technique. TEM images were acquired using JEOL J2100 as well as JEOL J2010F microscopes, both operated at an accelerating voltage of $200 \mathrm{kV}$. Transport Measurements: Electrical resistivity was determined by Van der Pauw method on unpatterned films, and Hall effect measurements were performed to determine carrier density and mobility. Measurements were performed in a PPMS Quantum Design system with magnetic fields up to $9 \mathrm{~T}$.

Optical Characterization: Variable-angle spectroscopic ellipsometry (VASE) measurements were carried in reflection mode at ambient conditions using a SOPRALAB GES5E ellipsometer. The ellipsometric spectra $(\Delta, \Psi)$ were recorded in the $230-990 \mathrm{~nm}$ spectral range at incident angles $\theta_{\mathrm{i}}$ of $\left.60^{\circ}-75^{\circ}\right)$. Optimized conditions were found to be $\theta_{\mathrm{i}}=65^{\circ}$ for $\mathrm{LAO}$, LSAT and NGO and $\theta_{\mathrm{i}}=68^{\circ}$ for STO. Data sets were analysed using WinElli II ${ }^{\odot}$ software. The SE data of the substrates were fitted first. Then, using the structure air/film/bulksubstrate, dielectric and optical coefficients $\left(\varepsilon=\varepsilon_{1}+i \varepsilon_{2}\right.$ and $\left.\tilde{n}=n+i \kappa\right)$ of the films were obtained (assuming isotropic $\varepsilon$ ). Films thickness was previously determined by XRR and inserted in the structure.

Fourier transform infrared reflectometry (FTIR) measurements were performed using a Hyperion 2000 (Bruker) microscope.

Synchrotron Experiments: X-ray absorption (XAS) was measured at the V-L2,3 edges at $300 \mathrm{~K}$ using linearly (either horizontal or vertical) polarized light and probed the X-ray linear 
dichroism (XLD) as the difference between the two light polarizations. To get access to the difference in orbital occupancy of $\mathrm{t}_{2 \mathrm{~g}}-(\mathrm{xy}$ and $(\mathrm{xz}, \mathrm{yz}))$ states, XAS spectra have to be taken for $\boldsymbol{E} / / a b$ and for $\boldsymbol{E} / / c$ (later shortened as $E_{a b}$ and $E_{c}$, respectively, where $a b$ and $c$ indicate inplane and out-of-plane X-ray electric field $\boldsymbol{E}$ directions, respectively). Due to geometrical constraints, following common practice, we collected the spectra in grazing incidence with the X-rays incidence direction $\boldsymbol{k}$ at $\theta=60^{\circ}$ with respect to the surface normal. The photocurrent was measured in the total electron yield (TEY) mode. Average XAS spectra were obtained by averaging the intensities of both linear polarizations (parallel to the surface normal $I\left(E_{c}\right)$ and perpendicular to it $\left.I\left(E_{a b}\right)\right)$ as $I_{0}=\left[I\left(E_{c}\right)+I\left(E_{a b}\right)\right] / 2$. The XLD signal was plotted as $I\left(E_{a b}\right)-I\left(E_{c}\right)$. The XAS experiments were performed at BOREAS beamline ${ }^{[43]}$ of ALBA synchrotron.

\section{Acknowledgements}

Financial support from the Spanish Ministry of Science, Innovation, and Universities, through the "Severo Ochoa" Programme for Centres of Excellence in R\&D (SEV-2015-0496) and the MAT2017-85232-R and MAT2016-79455-P (AEI/FEDER, EU) and from Generalitat de Catalunya (2017 SGR 1377 and 2017 SGR 776) is acknowledged. M.M. was financially supported by the Spanish Ministry of Science, Innovation, and Universities and this work has been done as a part of his Ph.D. program in Physics at Universitat Autònoma de Barcelona. The authors are very grateful to Dr. Mariona Coll for her advices on ellipsometry measurements and data treatment.

Received: ((will be filled in by the editorial staff))

Revised: ((will be filled in by the editorial staff)) Published online: ((will be filled in by the editorial staff))

\section{References}

[1] J. Heber, Nature 2009, 459, 28.

[2] D. G. Schlom, L. N. Pfeiffer, Nat. Mater. 2010, 9, 881.

[3] A. Ohtomo, H. Y. Hwang, Nature 2004, 427, 423. 
[4] X. Luo, Y. S. Oh, A. Sirenko, P. Gao, T. A. Tyson, K. Char, S.-W. Cheong, Appl. Phys. Lett. 2012, 100, 172112.

[5] H. Joon Kim, U. Kim, H. Min Kim, T. Hoon Kim, H. Sik Mun, B.-G. Jeon, K. Taek Hong, W.-J. Lee, C. Ju, K. Hoon Kim, K. Char, Appl. Phys. Express 2012, 5, 061102.

[6] H. J. Kim, U. Kim, T. H. Kim, J. Kim, H. M. Kim, B. G. Jeon, W. J. Lee, H. S. Mun, K. T. Hong, J. Yu, K. Char, K. H. Kim, Phys. Rev. B 2012, 86, 165205.

[7] S. Raghavan, T. Schumann, H. Kim, J. Y. Zhang, T. A. Cain, S. Stemmer, APL Mater. 2016, 4, 016106.

[8] S. M. Sze, K. Ng Kwok, Physics of Semiconductor Devices, John Wiley \& Sons, Hoboken, New Jersey, 2007.

[9] L. Zhang, Y. Zhou, L. Guo, W. Zhao, A. Barnes, H.-T. Zhang, C. Eaton, Y. Zheng, M. Brahlek, H. F. Haneef, N. J. Podraza, M. H. W. Chan, V. Gopalan, K. M. Rabe, R. Engel-Herbert, Nat. Mater. 2015, 15, 204.

[10] C. Eaton, J. A. Moyer, H. M. Alipour, E. D. Grimley, M. Brahlek, J. M. LeBeau, R. Engel-Herbert, J. Vac. Sci. Technol. A 2015, 33, 061504.

[11] M. Brahlek, L. Zhang, C. Eaton, H. T. Zhang, R. Engel-Herbert, Appl. Phys. Lett. 2015, $107,143108$.

[12] J. A. Moyer, C. Eaton, R. Engel-Herbert, Adv. Mater. 2013, 25, 3578.

[13] J. Wang, G. Rijnders, G. Koster, Appl. Phys. Lett. 2018, 113, 223103.

[14] W. C. Sheets, B. Mercey, W. Prellier, Appl. Phys. Lett. 2007, 91, 192102.

[15] B. Bérini, V. Demange, M. Bouttemy, E. Popova, N. Keller, Y. Dumont, A. Fouchet, Adv. Mater. Interfaces 2016, 3, 1600274.

[16] A. Boileau, A. Cheikh, A. Fouchet, A. David, R. Escobar-Galindo, C. Labbé, P. Marie, F. Gourbilleau, U. Lüders, Appl. Phys. Lett. 2018, 112, 021905.

[17] A. Fouchet, M. Allain, B. Bérini, E. Popova, P. E. Janolin, N. Guiblin, E. Chikoidze, J. Scola, D. Hrabovsky, Y. Dumont, N. Keller, Mater. Sci. Eng. B 2016, 212, 7. 
[18] A. Fouchet, J. E. Rault, M. Allain, B. Bérini, J. P. Rueff, Y. Dumont, N. Keller, J. Appl. Phys. 2018, 123, 055302.

[19] K. Yoshimatsu, T. Okabe, H. Kumigashira, S. Okamoto, S. Aizaki, A. Fujimori, M. Oshima, Phys. Rev. Lett. 2010, 104, 147601.

[20] D. H. Kim, D. W. Kim, B. S. Kang, T. W. Noh, D. R. Lee, K. B. Lee, S. J. Lee, Solid State Commun. 2000, 114, 473.

[21] D.-W. Kim, D. H. Kim, T. W. Noh, K. Char, J.-H. Park, K.-B. Lee, H.-D. Kim, J. Appl. Phys. 2000, 88, 7056.

[22] M. Kobayashi, K. Yoshimatsu, T. Mitsuhashi, M. Kitamura, E. Sakai, R. Yukawa, M. Minohara, A. Fujimori, K. Horiba, H. Kumigashira, Sci. Rep. 2017, 7, 16621.

[23] M. P. Wells, B. Zou, B. G. Doiron, R. Kilmurray, A. P. Mihai, R. F. M. Oulton, P. Gubeljak, K. L. Ormandy, G. Mallia, N. M. Harrison, L. F. Cohen, S. A. Maier, N. M. N. Alford, P. K. Petrov, Adv. Opt. Mater. 2017, 5, 1700622.

[24] D. Y. Wan, Y. L. Zhao, Y. Cai, T. C. Asmara, Z. Huang, J. Q. Chen, J. Hong, S. M. Yin, C. T. Nelson, M. R. Motapothula, B. X. Yan, D. Xiang, X. Chi, H. Zheng, W. Chen, R. Xu, Ariando, A. Rusydi, A. M. Minor, M. B. H. Breese, M. Sherburne, M. Asta, Q.-H. Xu, T. Venkatesan, Nat. Commun. 2017, 8, 15070.

[25] G. Sclauzero, K. Dymkowski, C. Ederer, Phys. Rev. B 2016, 94, 245109.

[26] H. Makino, I. Inoue, M. J. Rozenberg, I. Hase, Y. Aiura, S. Onari, Phys. Rev. B Condens. Matter Mater. Phys. 1998, 58, 4384.

[27] I. H. Inoue, O. Goto, K. Makino, H.Maiti, N. E. Hussey, M. Ishikawa, Phys. Rev. B Condens. Matter Mater. Phys. 1998, 58, 4372.

[28] A. Boileau, A. Cheikh, A. Fouchet, A. David, C. Labbé, P. Marie, F. Gourbilleau, U. Lüders, Adv. Opt. Mater. 2019, 7, 1801516.

[29] M. Mirjolet, F. Sánchez, J. Fontcuberta, Adv. Funct. Mater. 2019, 29, 1808432.

[30] K. Ganguly, A. Prakash, B. Jalan, C. Leighton, APL Mater. 2017, 5, 056102. 
[31] M. Kobayashi, K. Yoshimatsu, E. Sakai, M. Kitamura, K. Horiba, A. Fujimori, H. Kumigashira, Phys. Rev. Lett. 2015, 115, 076801.

[32] J. R. Petrie, C. Mitra, H. Jeen, W. S. Choi, T. L. Meyer, F. A. Reboredo, J. W. Freeland, G. Eres, H. N. Lee, Adv. Funct. Mater. 2016, 26, 1564.

[33] K. Ellmer, Nat. Photonics 2012, 6, 809.

[34] S. Stemmer, A. J. Jacobson, X. Chen, A. Ignatiev, J. Appl. Phys. 2001, 90, 3319.

[35] J. Gazquez, W. Luo, M. P. Oxley, M. Prange, M. A. Torija, M. Sharma, C. Leighton, S. T. Pantelides, S. J. Pennycook, M. Varela, Nano Lett. 2011, 11, 973.

[36] J. Gazquez, S. Bose, M. Sharma, M. A. Torija, S. J. Pennycook, C. Leighton, M. Varela, APL Mater. 2013, 1, 012105.

[37] L. López-Conesa, J. M. Rebled, D. Pesquera, N. Dix, F. Sánchez, G. Herranz, J. Fontcuberta, C. Magén, M. J. Casanove, S. Estradé, F. Peiró, Phys. Chem. Chem. Phys. 2017, 19, 9137.

[38] D. Pesquera, G. Herranz, A. Barla, E. Pellegrin, F. Bondino, E. Magnano, F. Sánchez, J. Fontcuberta, Nat. Commun. 2012, 3, 1189.

[39] J. Stöhr, NEXAFS Spectroscopy, Springer-Verlag, Berlin, 1992.

[40] X. W. Lin, Y. Y. Wang, V. P. Dravid, P. M. Michalakos, M. C. Kung, Phys. Rev. B 1993, $47,3477$.

[41] M. Wu, J.-C. Zheng, H.-Q. Wang, Phys. Rev. B 2018, 97, 245138.

[42] B. L. Chamberland, P. S. Danielson, J. Solid State Chem. 1971, 3, 243.

[43] A. Barla, J. Nicolás, D. Cocco, S. M. Valvidares, J. Herrero-Martín, P. Gargiani, J. Moldes, C. Ruget, E. Pellegrin, S. Ferrer, J. Synchrotron Radiat. 2016, 23, 1507. 

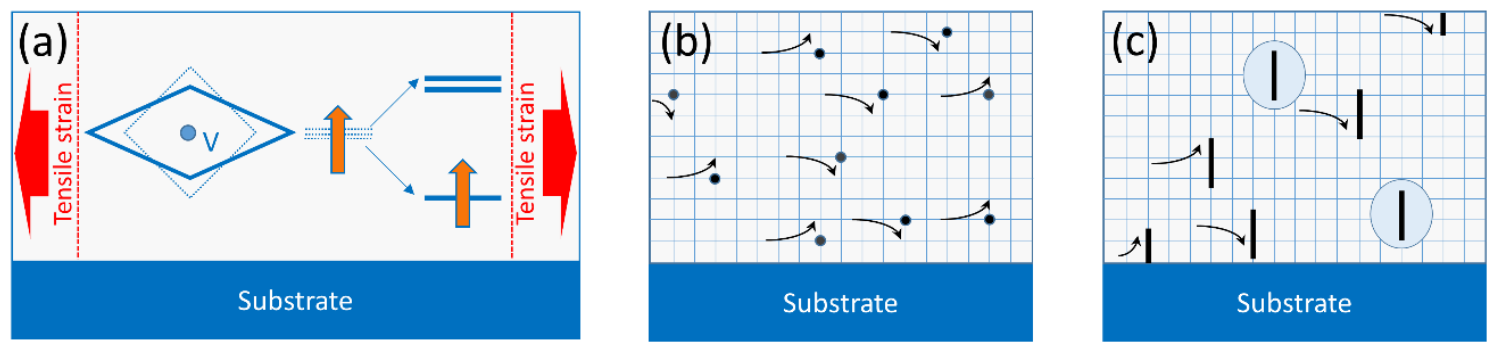

Figure 1. a) Sketch of the impact of (tensile) strain on the $\mathrm{VO}_{6}$ polyhedron deformation and breaking of degeneracy of $3 \mathrm{~d}^{1}-\mathrm{t}_{2 \mathrm{~g}}(\mathrm{xy}, \mathrm{xz}, \mathrm{yz})$ orbitals. b) Carrier scattering (curved arrows) caused by point defects. c) Carrier scattering caused by extended defects, such as dislocations and cracks. Charge-screened defects are indicated by circles.

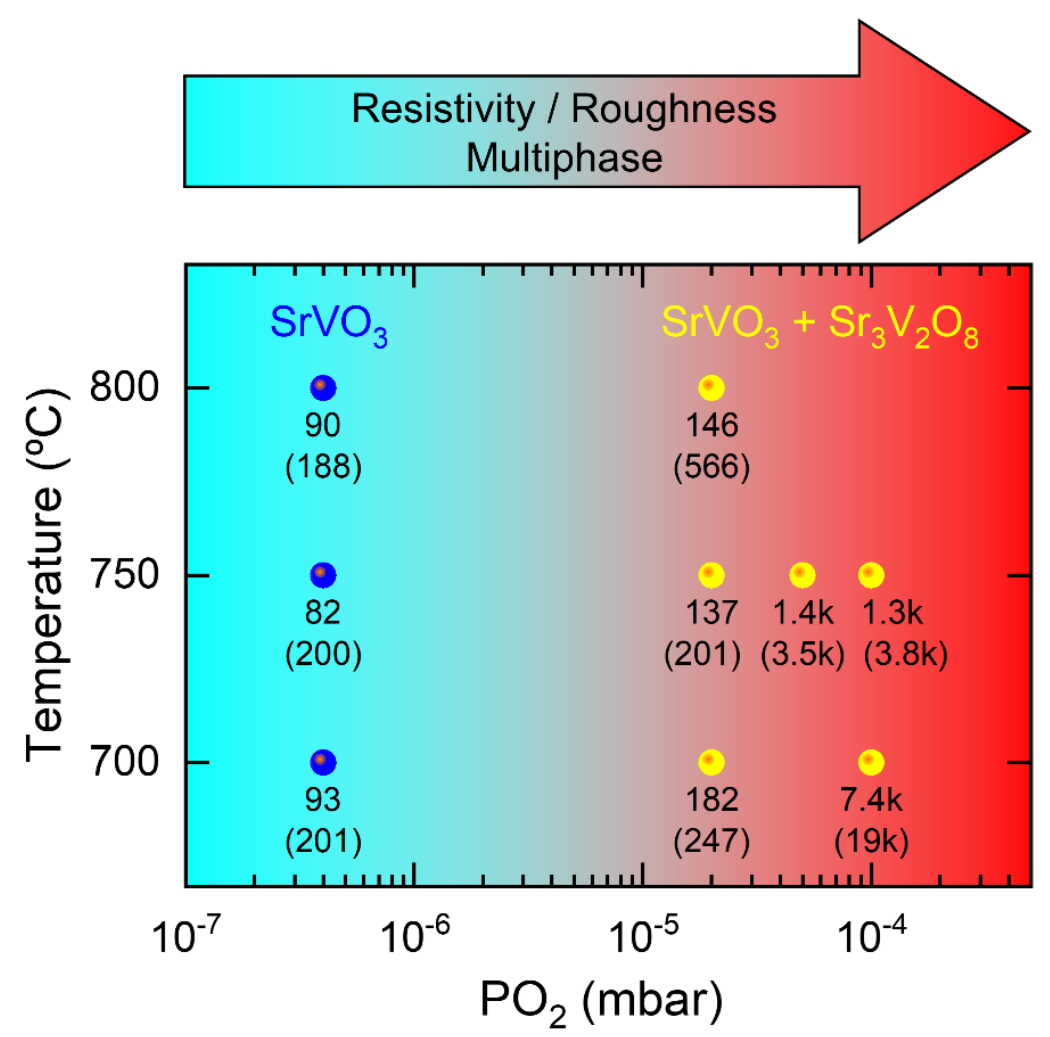

Figure 2. Growth window describing the crystalline phases and electrical properties of SVO films grown on (001) LSAT and (001) STO substrates, as a function of $\mathrm{PO}_{2}$ and T during growth. The indicated numbers are the room-temperature resistivity data (in $\mu \Omega \mathrm{cm}$ ) for SVO on LSAT. Within parenthesis are the corresponding data for SVO films on STO. The color scale (from cyan to red) illustrates the increasing resistivity and roughness of the films. 

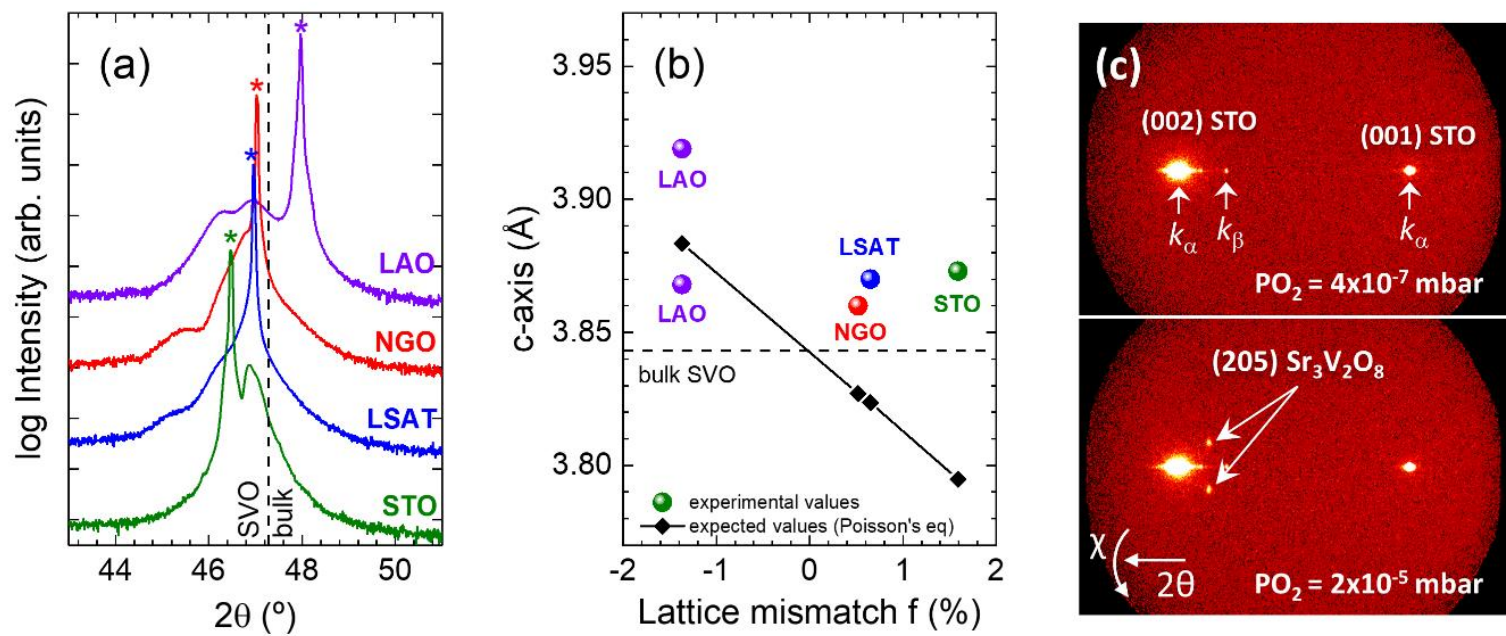

Figure 3. a) XRD $\theta-2 \theta$ scans of films grown at $\mathrm{PO}_{2}=4 \times 10^{-7} \mathrm{mbar}$ and $\mathrm{T}=750^{\circ} \mathrm{C}$ on different substrates. The (002) reflection of the substrate is indicated by a star. The spectra are vertically shifted for clarity. b) Out-of-plane cell parameters (c-axis) of the films as a function of the lattice mismatch with the substrate (circles). Diamonds represent the expected c-axis values for fully strained films, evaluated using Poisson's equation and assuming unit cell volume conservation. c) $2 \theta-\chi$ frames of SVO films on $\mathrm{STO}$ at $\mathrm{T}=750^{\circ} \mathrm{C}$ and $\mathrm{PO}_{2}=4 \times 10^{-7}$ mbar (upper frame) and $\mathrm{PO}_{2}=2 \times 10^{-5} \mathrm{mbar}$ (lower frame).
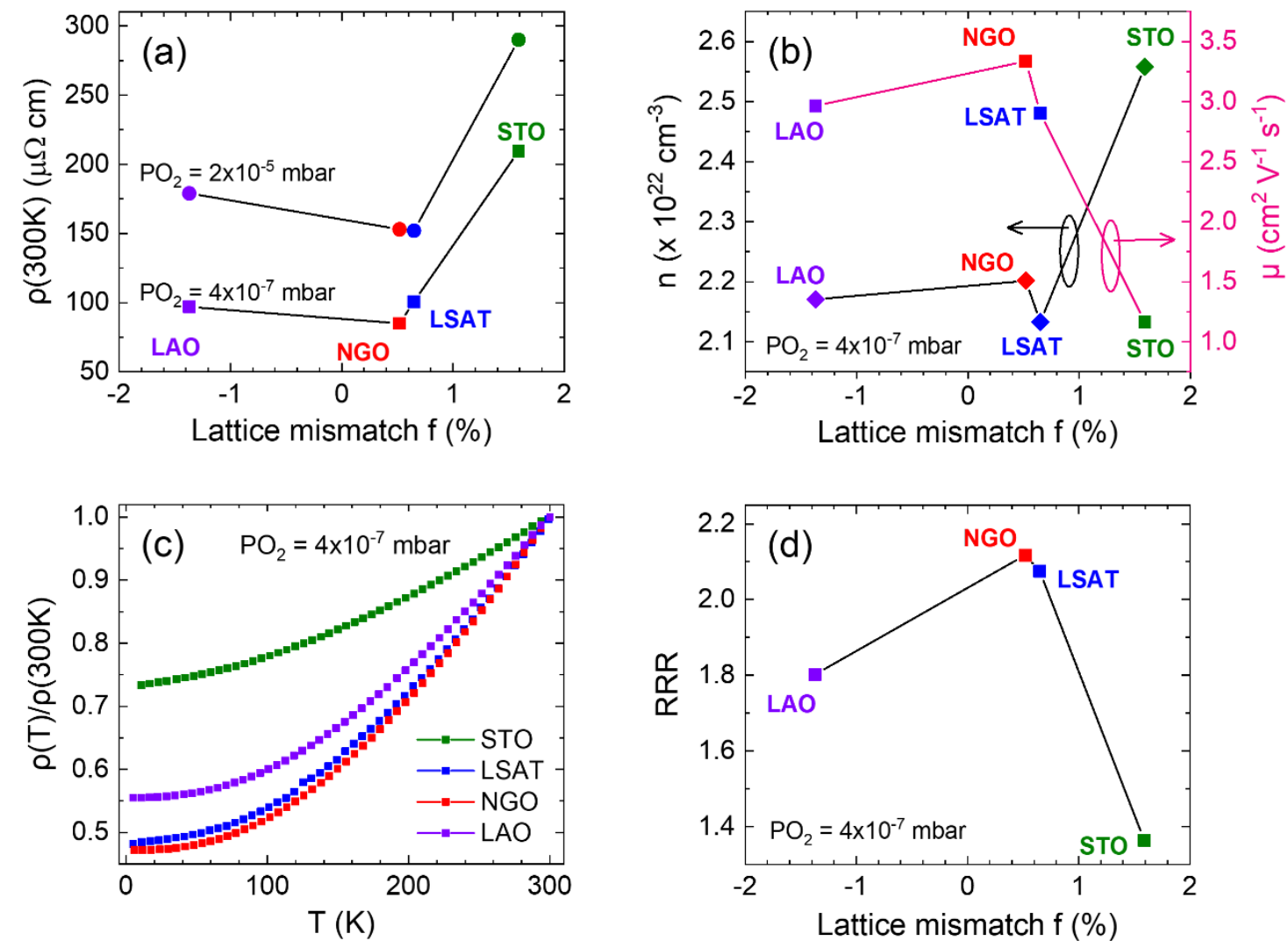

Figure 4. a) Room-temperature resistivity $\rho(300 \mathrm{~K})$ of films deposited on different substrates at $\mathrm{PO}_{2}=4 \times 10^{-7} \mathrm{mbar}$ (squares) and $\mathrm{PO}_{2}=2 \times 10^{-5} \mathrm{mbar}$ (circles). b) Carrier density (diamonds) and carrier mobility (squares) of the films grown at the lowest pressure $\left(\mathrm{PO}_{2}=4 \times 10^{-7} \mathrm{mbar}\right)$.

c) Temperature dependence of the normalized resistivity $\rho(T) / \rho(300 \mathrm{~K})$ of the same films 
grown at the lowest pressure $\left(\mathrm{PO}_{2}=4 \times 10^{-7} \mathrm{mbar}\right)$; the corresponding structural mismatch is indicated. d) Residual resistivity ratio $(\mathrm{RRR}=\rho(300 \mathrm{~K}) / \rho(5 \mathrm{~K}))$.

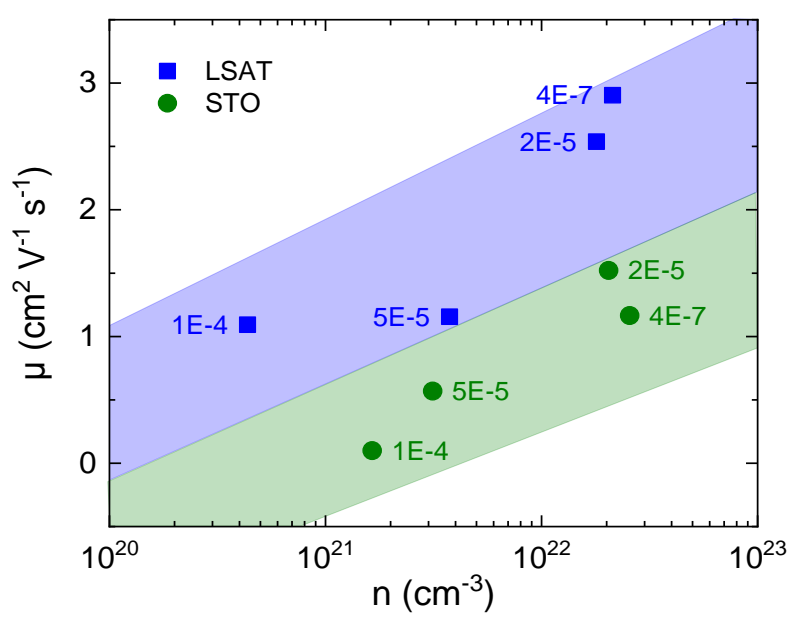

Figure 5. Carrier mobility versus carrier density for SVO//LSAT (squares) and SVO//STO (circles) films deposited at various $\mathrm{PO}_{2}$.
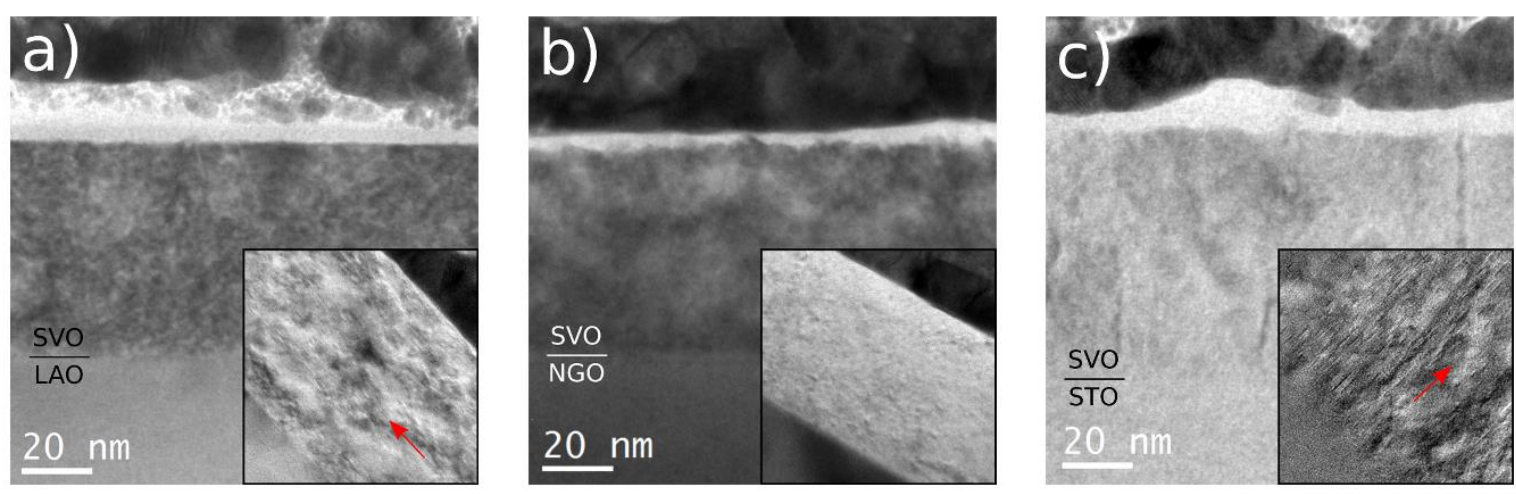

Figure 6. Cross-section bright field TEM images of the SVO films grown on a) LAO, b) NGO, and c) STO substrates. Insets: bright field TEM images obtained by tilting the crystals to obtain two-beam conditions, highlighting the presence of defects in particular directions. 

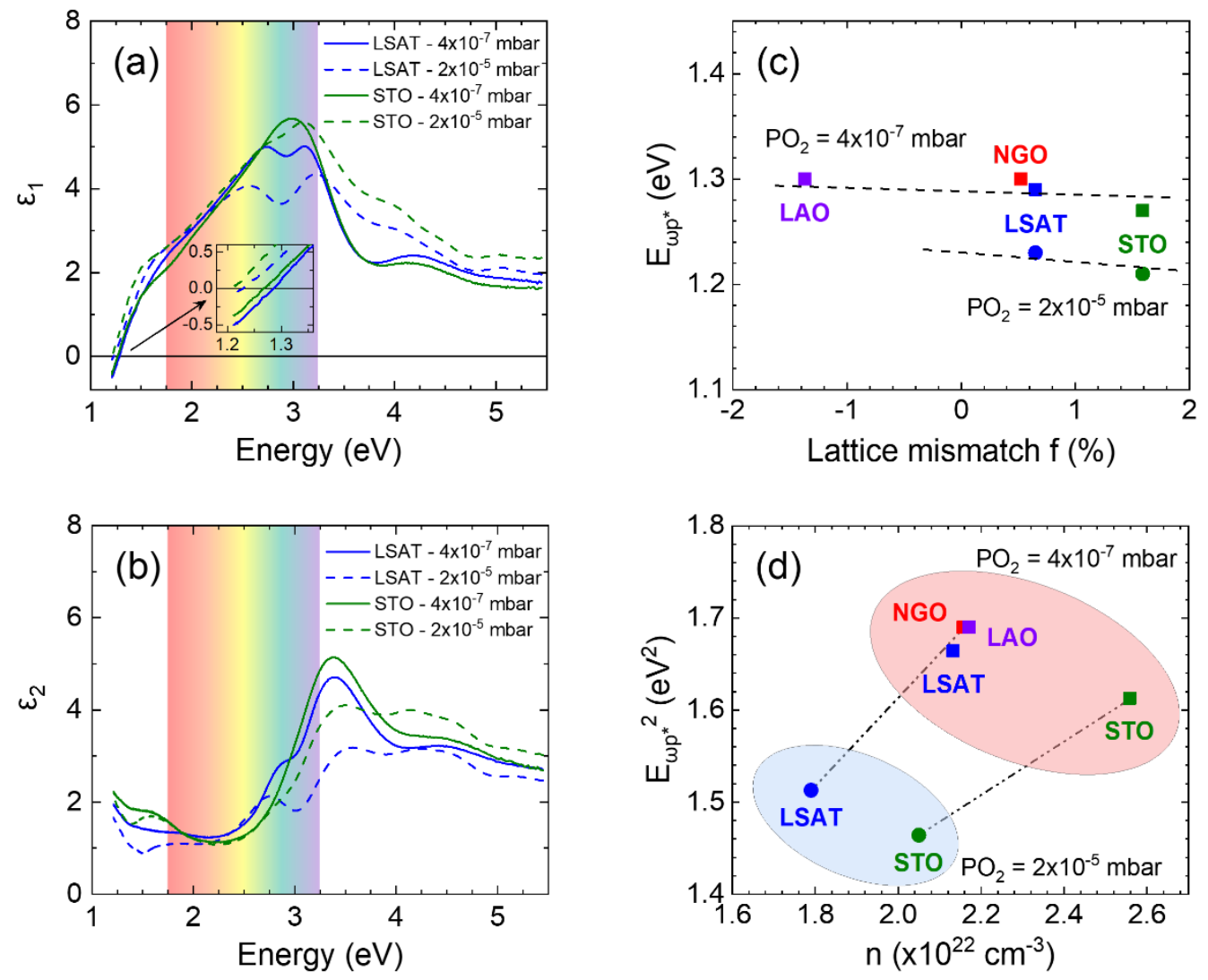

Figure 7. a,b) Complex permittivity $\varepsilon=\varepsilon_{1}+\mathrm{i} \varepsilon_{2}$ of SVO//LSAT and SVO//STO films grown at $\mathrm{PO}_{2}=4 \times 10^{-7}$ mbar and $\mathrm{PO}_{2}=2 \times 10^{-5}$ mbar, as indicated. c) Dependence of the measured screened plasma energy $\left(\mathrm{E}_{\omega \mathrm{p}^{*}}=\hbar \omega_{\mathrm{p}}{ }^{*}\right)$ on the substrate mismatch $\mathrm{f}$. The lines through the data are guides for the eye. d) Dependence of $\mathrm{E}_{\omega \mathrm{p}} *^{2} v s$ carrier concentration $\mathrm{n}$ as derived from Hall measurements (Figure 4). The lines through the data allows visualizing the modulation of $\mathrm{E}_{\omega \mathrm{p}}{ }^{2}(\mathrm{n})$ with growth conditions and the distinctive behavior of SVO//STO. Encircled samples where grown under the same $\mathrm{PO}_{2}$. 

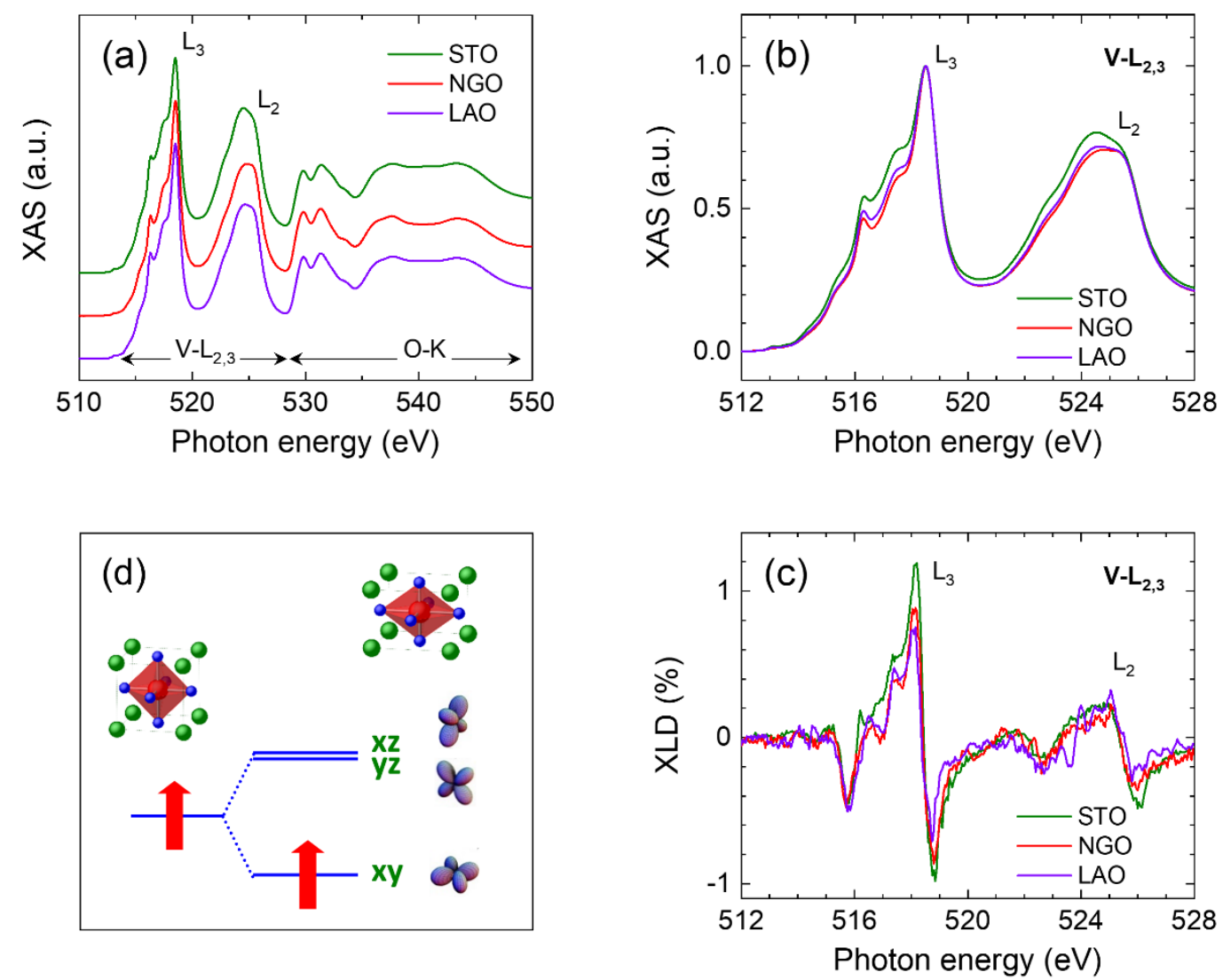

Figure 8. a) X-ray absorption (XAS) spectra at V-L $\mathrm{L}_{2,3}$ and $\mathrm{O}-\mathrm{K}$ edges for SVO films (70-80 $\mathrm{nm}$ thick) grown on STO, NGO and LAO substrates, as indicated. b) A zoom of the XAS spectra around the $\mathrm{V}-\mathrm{L}_{2,3}$ edges. c) Corresponding X-ray linear dichroism (XLD) (\% of white line intensity) spectra of the same samples. d) Sketch of the V-3d- $t_{2 g}$ band splitting resulting from in-plane tensile strain of the $\mathrm{VO}_{6}$ octahedron. 
Early transition metal oxides (e.g. SrVO3) may be the pillar for a new generation of transparent conductors. However, growth of thin films faces severe constrains associated to structural mismatch with substrates and harsh conditions required to get optimal conductivity. It is shown that electrical conductivity can be tuned while preserving optical transparency in the visible, alleviating obstacles for their integration.

\section{Keyword: transparent conducting oxides}

M. Mirjolet, H. B. Vasili, L. López-Conesa, S. Estradé, F. Peiró, J. Santiso, F. Sánchez, P. Machado, P. Gargiani, M. Valvidares and J. Fontcuberta*

Independent Tuning of Optical Transparency Window and Electrical Properties of Epitaxial SrVO3 Thin Films by Substrate Mismatch

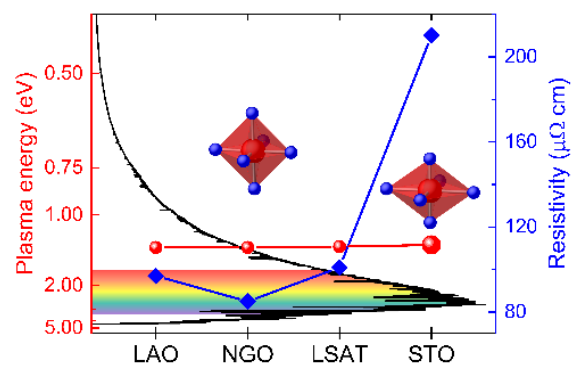




\section{Supporting Information}

\section{Independent Tuning of Optical Transparency Window and Electrical Properties of}

Epitaxial SrVO3 Thin Films by Substrate Mismatch

Mathieu Mirjolet, Hari Babu Vasili, LLuís López-Conesa, Sònia Estradé, Francesca Peiró, José Santiso, Florencio Sánchez, Pamela Machado, Pierluigi Gargiani, Manuel Valvidares and Josep Fontcuberta*

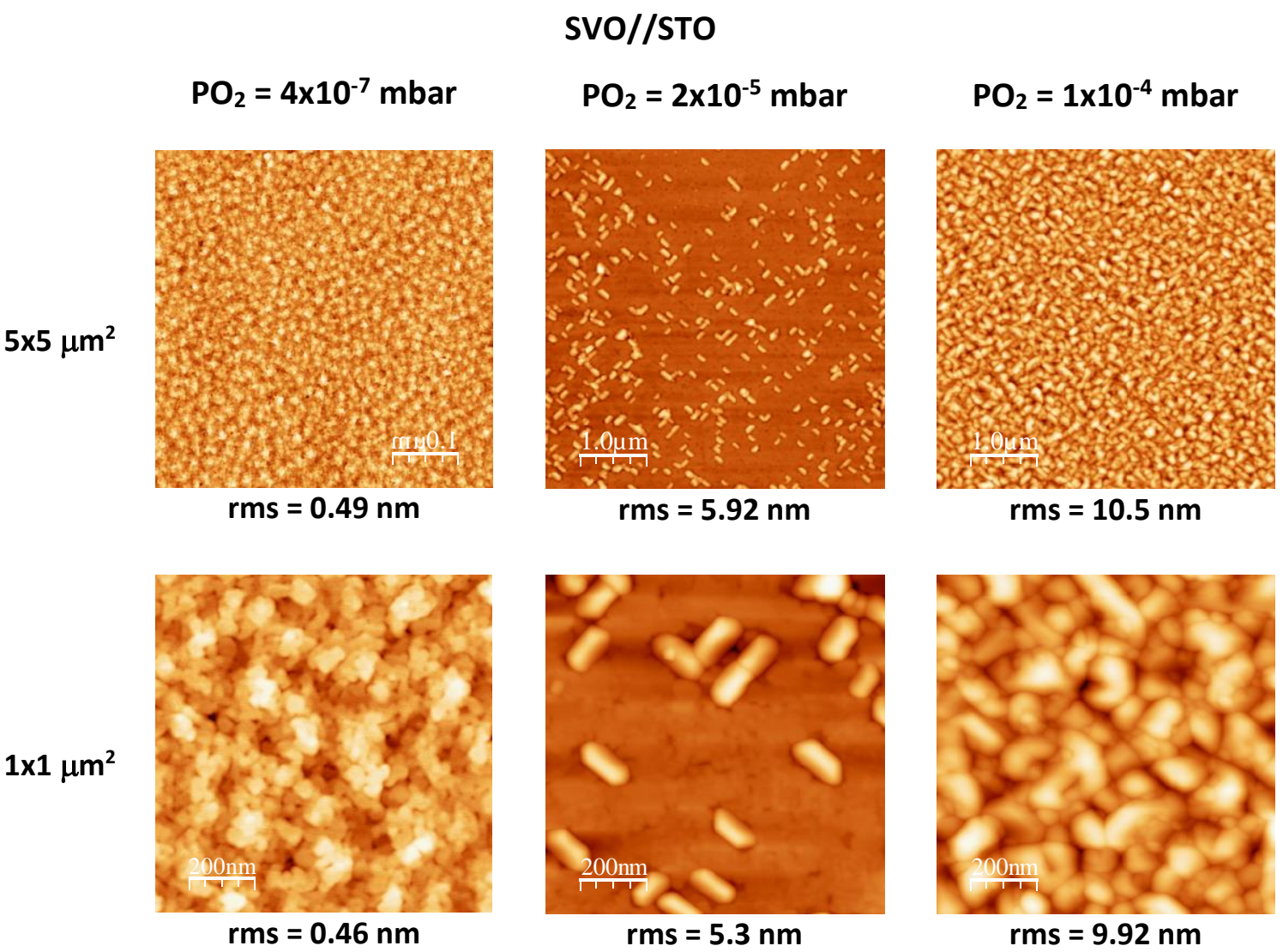

Figure S1. AFM topographic images, $5 \mu \mathrm{m}$ x $5 \mu \mathrm{m}$ in size (top images), of SVO films grown on STO substrates at $750^{\circ} \mathrm{C}$ and various $\mathrm{PO}_{2}$ (as indicated). Bottom images are $1 \mu \mathrm{mx} 1 \mu \mathrm{m}$ zooms emphasizing the outgrowths formed at film surface when deposition is performed at $\mathrm{PO}_{2}>4 \times 10^{-7}$ mbar. The root-mean square (rms) roughness is indicated below each image. 


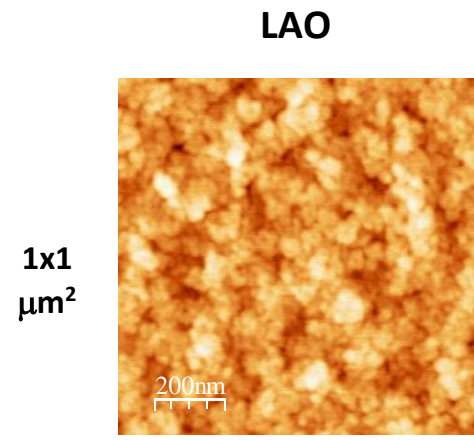

$\mathrm{rms}=0.48 \mathrm{~nm}$

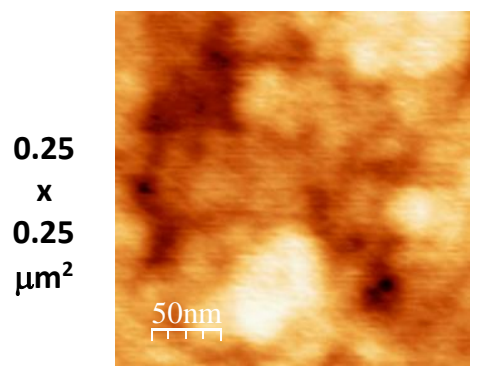

$\mathrm{rms}=0.51 \mathrm{~nm}$

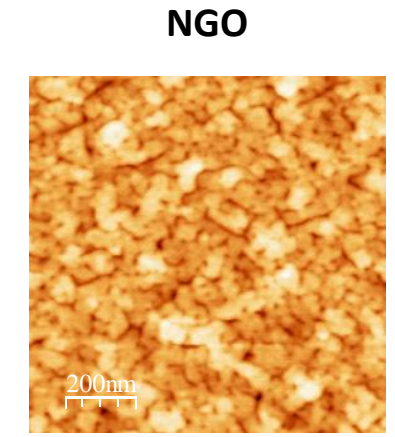

$\mathrm{rms}=0.46 \mathrm{~nm}$

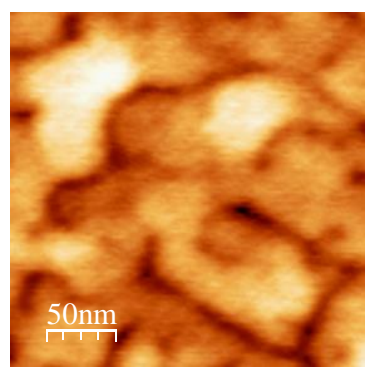

$\mathrm{rms}=0.46 \mathrm{~nm}$
LSAT

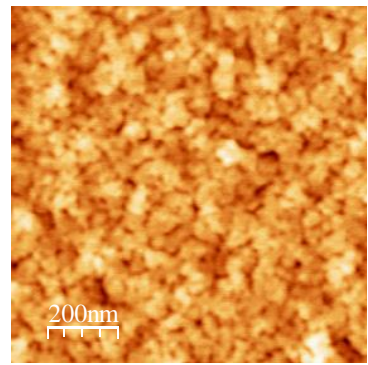

$\mathrm{rms}=0.41 \mathrm{~nm}$

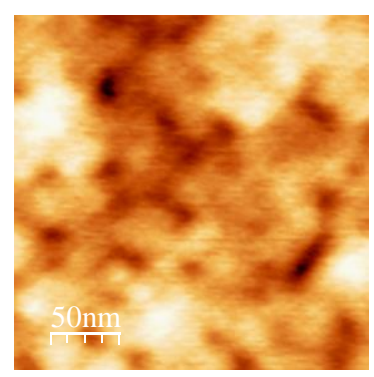

$\mathrm{rms}=0.44 \mathrm{~nm}$
STO

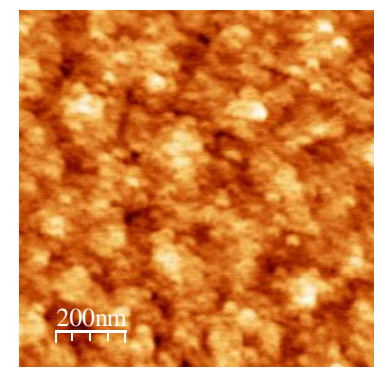

$\mathrm{rms}=0.52 \mathrm{~nm}$

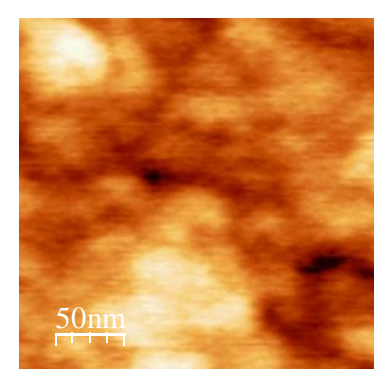

$\mathrm{rms}=0.5 \mathrm{~nm}$

Figure S2. AFM topographic images, $1 \mu \mathrm{m} \times 1 \mu \mathrm{m}$ in size (top images), of SVO films grown at $\mathrm{PO}_{2}=4 \times 10^{-7}$ mbar and $\mathrm{T}=750^{\circ} \mathrm{C}$, on various substrates. Bottom images are $250 \mathrm{~nm} \times 250 \mathrm{~nm}$ zooms emphasizing the granularity of the films. The root-mean square (rms) roughness is indicated below each image.

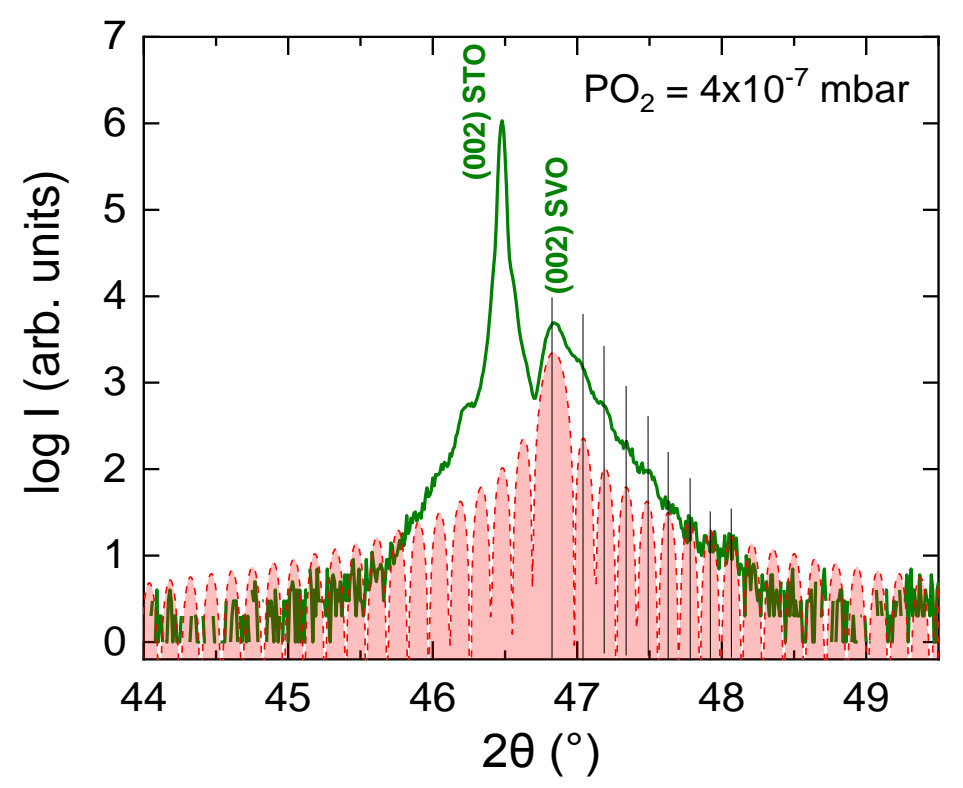

Figure S3. High resolution XRD $\theta-2 \theta$ scan of SVO//STO film grown at $\mathrm{PO}_{2}=4 \times 10^{-7} \mathrm{mbar}$ and $\mathrm{T}=750^{\circ} \mathrm{C}$. Laue fringes can be observed. The extracted thickness of $67 \mathrm{~nm}$ is in agreement with the one determined from X-ray reflectivity measurements $(70 \mathrm{~nm})$. 
(a)

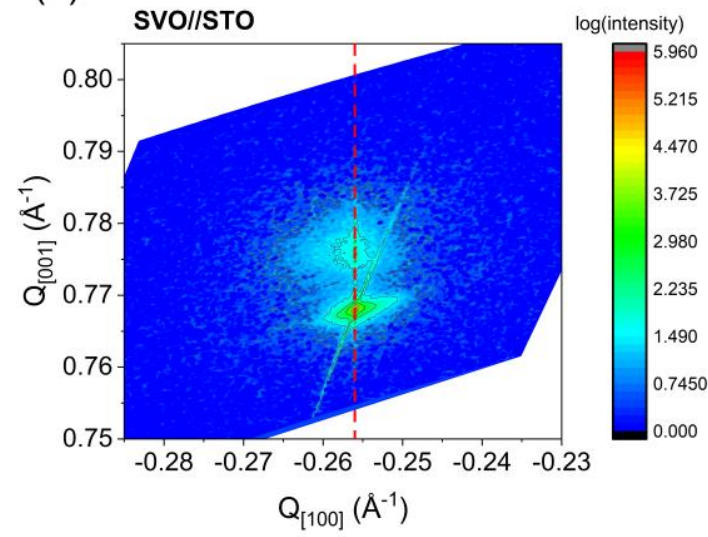

(c)

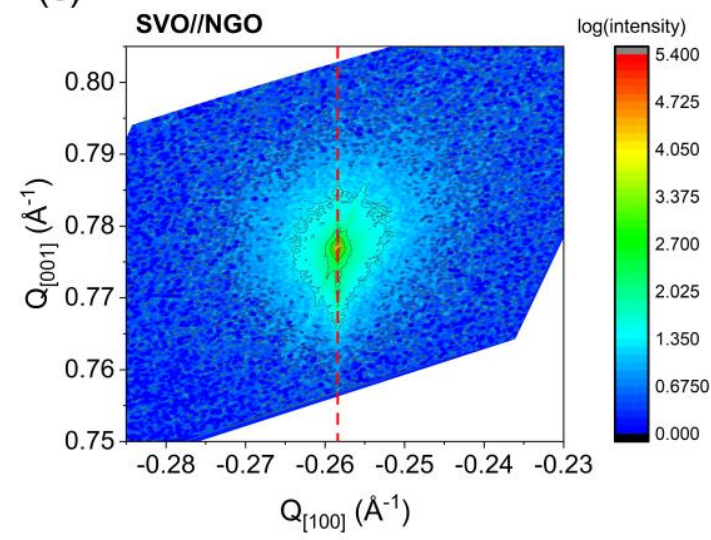

(b)

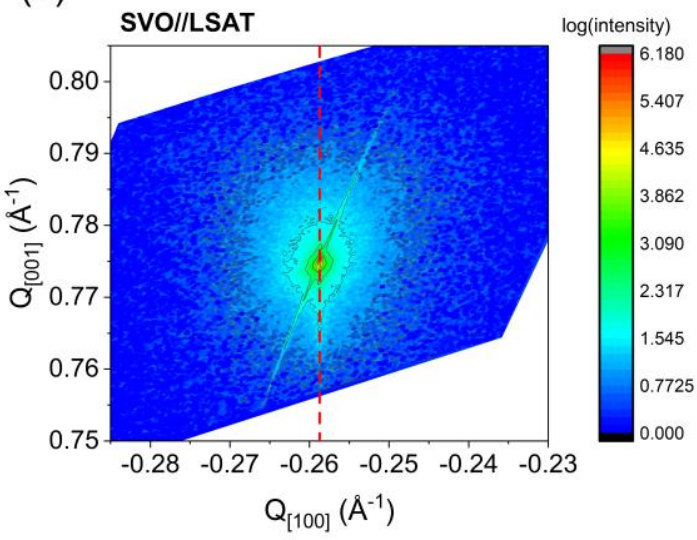

(d)

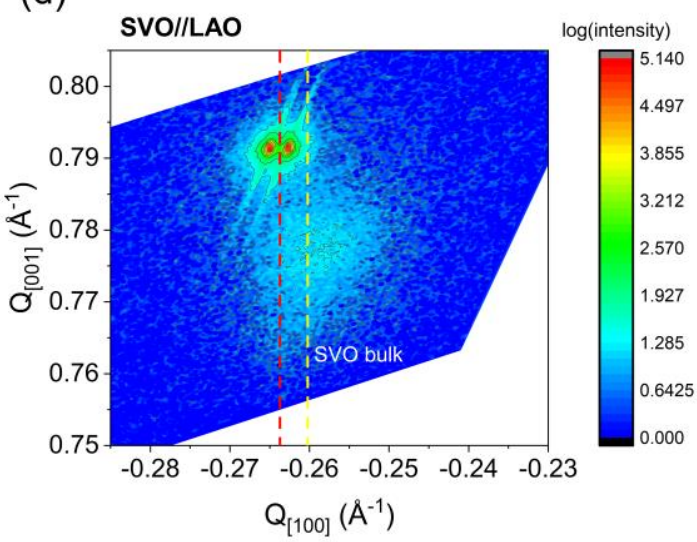

Figure S4. XRD reciprocal space maps around (-103) reflections of a) SVO//STO, b) $\mathrm{SVO} / / \mathrm{LSAT}$, c) SVO//NGO, and d) SVO//LAO. Notice in a), b) and c) that the in-plane cell parameter of the film coincides with that of the substrate (vertical red dashed line). This is not the case in d) where the center (vertical white dashed line) of the relatively broad SVO reflection is clearly shifted with respect to that of the substrate. 


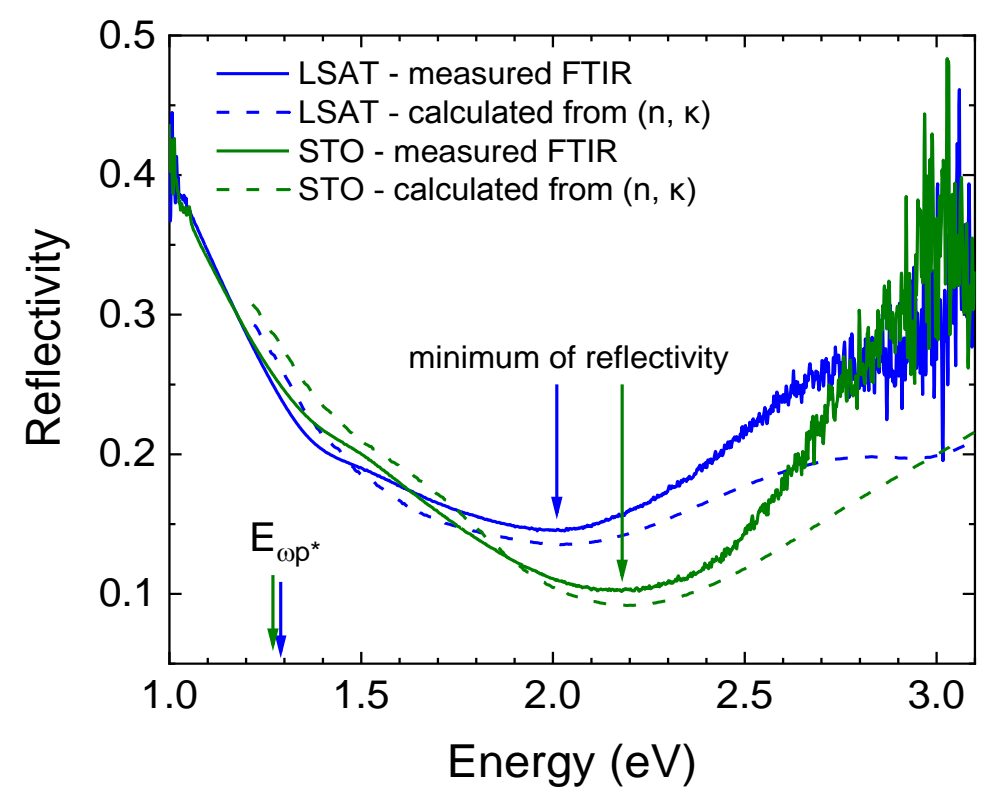

Figure S5. Calculated reflectivity and measured FTIR spectra at normal incidence, of $\mathrm{SVO} / / \mathrm{LSAT}$ and SVO//STO films grown at $\mathrm{PO}_{2}=4 \times 10^{-7} \mathrm{mbar}$ and $\mathrm{T}=750^{\circ} \mathrm{C}$. Reflectivity was calculated using a model consisting of a substrate $(0.5 \mathrm{~mm}$ thick) and the corresponding SVO layer thickness. The optical parameters $(n, \kappa)$ of film and substrate where obtained from the ellipsometry measurements as indicated. 